\title{
CONSTRUCTIONS IN COGNITIVE GRAMMAR
}

\author{
RONALD W. LANGACKER \\ University of California, San Diego
}

\begin{abstract}
Cognitive Grammar claims that grammatical structure is symbolic in nature and that constructions are the primary objects of description. A construction is an assembly of symbolic structures (form-meaning pairings) linked by correspondences. While constructions have certain characteristic features, various kinds of departure from this prototype are also common. Grammatical patterns are captured by constructional schemas (schematic symbolic assemblies). Grammatical dependencies reside in correspondences between semantic elements, with the consequence that constituency is non-essential and often variable. The symbolic view of grammar offers a viable alternative to the autonomy thesis.
\end{abstract}

Keywords: Cognitive Grammar, construction, grammatical dependencies, constituency, semantic composition

\section{Architecture}

More than one linguistic theorist has voiced the opinion that cognitive linguists, including myself, fail to recognize the existence of grammar. That is simply false. The question is not whether grammar exists - for it does - but rather, what is it like? My framework of Cognitive Grammar (CG) diverges from standard assumptions in two fundamental respects: (i) its claim that grammar is symbolic in nature; and (ii) its focus on constructions (rather than rules) as the primary objects of description (Langacker (1987a, 1990, 1991, 1999a)).

The first claim denies the autonomy of syntax. Crucially, though, we need to distinguish between two definitions of autonomy that have often been confused. By the first definition, syntax (and more generally, grammar) is autonomous unless it is fully predictable in terms of meaning and other independent factors. Let us call this weak autono$m y$. It implies that grammar does not just fall out or emerge automatically from other phenomena. Rather, it has to be specifically learned by children and explicitly described by linguists. Observe that

English Linguistics 20: 1 (2003) 41-83 - 41-

(C) 2003 by the English Linguistic Society of Japan 
weak autonomy says nothing about the nature of grammatical structure, only its non-predictability. The second definition says that grammar is autonomous by virtue of being distinct from both lexicon and semantics, constituting a separate level of representation whose description requires a special set of irreducible grammatical primitives. Let us call this strong autonomy.

All cognitive linguists accept weak autonomy. Grammar exists and has to be described as such. Only its nature and proper characterization are at issue. The basic claims of CG presuppose weak autonomy but constitute a radical alternative to strong autonomy. For one thing, CG holds that lexicon, morphology, and syntax form a continuum, divided only arbitrarily into discrete components. Moreover, it claims that lexicon and grammar are fully describable as assemblies of symbolic structures, where a symbolic structure is simply the pairing between a semantic structure and a phonological structure (its semantic and phonological poles). This has several consequences. First, grammar is not distinct from semantics, but rather incorporates semantics as one of its two poles. Second, grammatical description does not rely on special, irreducible grammatical primitives, but only on symbolic structures, each reducible to a form-meaning pairing. Third, every construct validly posited in grammatical description has a semantic pole and is therefore meaningful (though the meanings are often quite schematic).

Like Construction Grammar, CG takes constructions, rather than rules, to be the primary objects of grammatical description (Fillmore (1988); Fillmore, Kay, and O'Connor (1988); Goldberg (1995); Croft (2001); cf. Langacker (To appear b)). Grammar comprises regularities of varying degrees of generality - patterns that speakers internalize and that linguists need to discover and describe. What are these patterns like, and how can we best describe them? Three kinds of devices have commonly been employed in linguistic description: rules, filters, and schemas. These imply different kinds of relationships between specific expressions (e.g. sentences) and the patterns they manifest.

By rules, I mean constructive rules analogous to the phrase structure rules and transformations of classic generative syntax. What is important here is the notion that rules and expressions are quite different in nature and related only indirectly. It is only required that, through their cumulative application, some set of rules serve collectively to con- 
struct a given expression. Rules do not necessarily resemble the expressions they help derive. Filters are negative statements indicating that a particular configuration of elements is not permitted. By definition, filters are distinct from the expressions they help describe. Schemas bear the closest relation to expressions. They are templates for expressions, representing the abstracted commonality of sets of expressions parallel in certain respects. Schemas are thus directly analogous to the expressions they characterize apart from their level of specificity.

In CG, grammatical patterns are represented by means of schemas. A construction is defined as either an expression (of any size), or else a schema abstracted from expressions to capture their commonality (at any level of specificity). Expressions and the patterns they instantiate are thus the same in their basic nature, differing only in degree of specificity. Both specific expressions and abstracted schemas are capable of being entrenched psychologically and conventionalized in a speech community, in which case they constitute established linguistic units. Specific expressions with the status of units are traditionally recognized as lexical items. More schematic units correspond to what is traditionally regarded as grammar. The difference, though, is a matter of degree, and in CG these form a continuum. Every constructionwhether lexical or grammatical-is characterized as an assembly of symbolic structures.

$\mathrm{CG}$ is highly restrictive owing to the content requirement. The elements permitted in a linguistic description are limited to: (i) semantic, phonological, and symbolic structures that actually occur as (parts of) expressions; (ii) schematizations of permitted structures; and (iii) categorizing relationships between permitted structures. Thus the only elements ascribable to a linguistic system are those which are either part of the primary data (namely, occurring expressions), hence directly apprehended, or else emerge from the primary data by means of the basic psychological phenomena of schematization and categorization. Ruled out by the content requirement are such elements as filters, purely syntactic primitives (with neither semantic nor phonological content), and derivations from underlying structures.

Let us then consider what the content requirement does permit. Permitted first, as shown in (1), are semantic structures (abbreviated S) and phonological structures (P). These can be of any size and any degree of internal complexity. A symbolic structure $(\Sigma)$ consists in the linkage of a semantic and a phonological structure (its two poles). 
Symbolic structures combine with one another (in ways to be discussed) to form assemblies of symbolic structures, which can also be of any size and any degree of internal complexity. When these assemblies are specific (rather than schematic), they constitute expressions (E), such as words, phrases, clauses, etc. (When those expressions are entrenched and conventionalized, they are recognized as lexical items.)

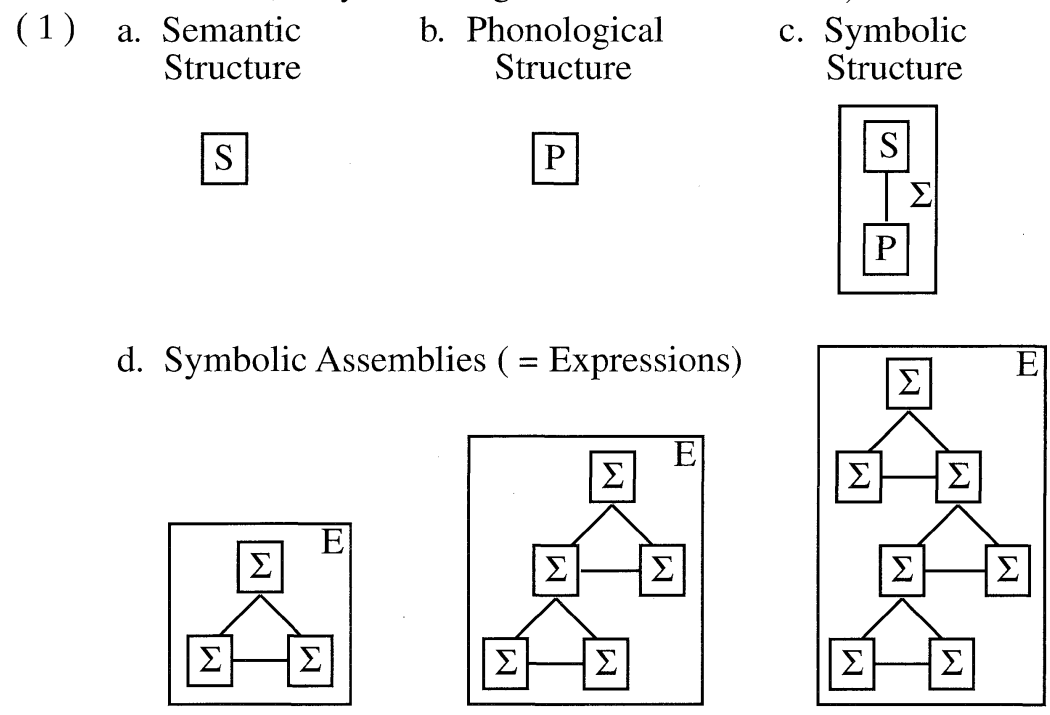

Permitted next, as shown in (2), are schemas (Sch). Each represents the abstracted commonality observable in sets of occurring expressions, or in schemas previously extracted. Schematization can be carried to whatever level of abstraction the data supports.

(2)

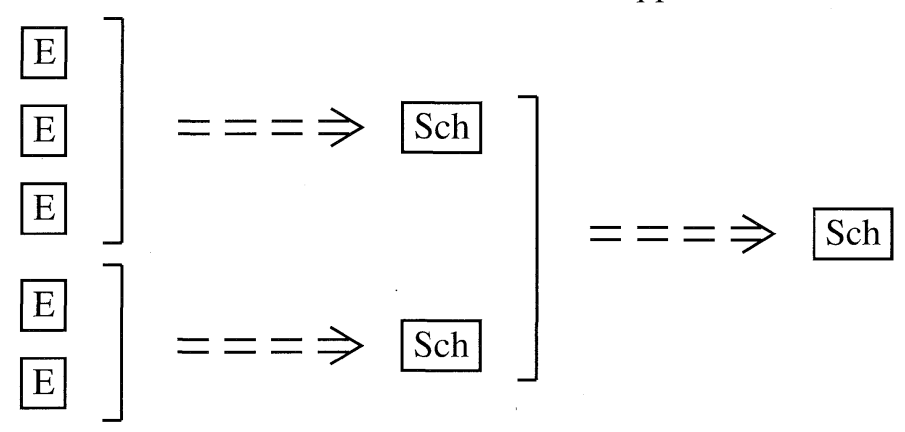

Also permitted are relationships of categorization, of which there are two basic sorts, described in (3). One sort is the relation between a schema and more specific structures in which the schema is immanent 
(i.e. observable without distortion). These more specific structures thus elaborate (or instantiate) the schema. For this I use a solid arrow. A dashed arrow represents extension, implying some conflict between the categorizing structure and the one it categorizes. In this case the categorizing structure can be regarded as a prototype (at least in local terms).

(3) a. Elaboration

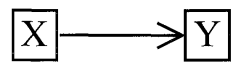

b. Extension

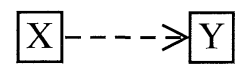

A linguistic system thus comprises vast networks of structures linked by categorizing relationships, as sketched in (4a). Included in such networks are specific expressions with the status of conventional units, as well as schemas representing various levels of abstraction (or schematicity). Of course, a particular expression - whether fixed or novel - is categorized simultaneously by many schemas, each corresponding to a particular facet of its structure. Collectively, the set of schemas which categorize it constitutes its structural description (i.e. its interpretation with respect to the linguistic system), as shown in (4b). The expression is well-formed (or grammatical) to the extent that these categorizations involve elaboration rather than extension.

(4) a. Categorization Network

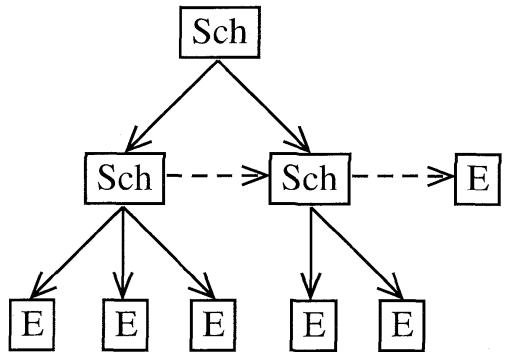

b. Structural Description

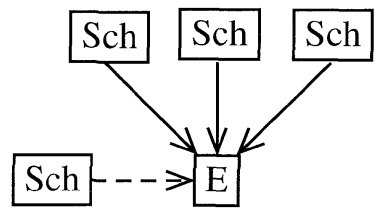

In this framework, grammatical patterns are captured by constructional schemas, i.e. schematic symbolic assemblies (Langacker (1987a: ch. 10, 1988a, 2000)). A constructional schema describes, in schematic terms, how simpler expressions combine to form a more complex expression. It can therefore function as a template guiding the formation of new expressions, and also serves to categorize the relevant facets of such expressions, as shown in (5). 


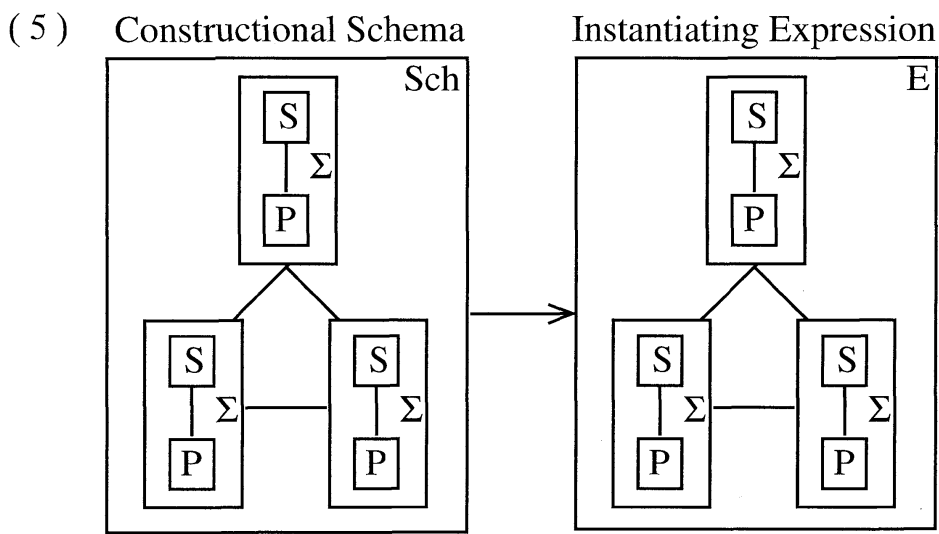

Why does grammar exist? There would be no need for grammatical patterns if a minimal symbolic structure (i.e. a morpheme) were available to symbolize every notion we might have occasion to express. That of course is not feasible because conceptualization is so flexible and open-ended. Grammar allows the formation of symbolically complex expressions capable of evoking novel conceptions of any degree of complexity. It does so by means of constructional schemas. Each such schema is a pattern for combining simpler symbolic structures to form more complex ones. As such, it specifies how their component elements are semantically integrated, and how they are phonologically integrated to symbolize their semantic integration. Consequently, patterns of semantic composition can be identified as the semantic poles of constructional schemas. Semantic composition is not distinct from grammar, but constitutes the semantic pole of grammar (just as lexical meanings are not distinct from lexical items, but constitute their semantic poles). These patterns of semantic composition do not completely determine the meanings of complex expressions (Langacker (To appear a)). Here, though, I will concentrate on grammatical constructions and the compositional aspects of linguistic meaning captured by constructional schemas.

\section{Basic Semantic Notions}

To describe in detail the CG view of grammatical constructions, I must first introduce some basic notions pertaining to semantic structure. In cognitive semantics, meaning is identified with conceptualization, in the broadest sense. Pivotal to linguistic semantics is our ability to 
construe the same situation in alternate ways (Langacker (1993a)). Among the dimensions of construal are the level of specificity at which a situation is characterized, the perspective adopted for viewing it, and the degree of prominence conferred on the elements within it.

By specificity (or conversely, schematicity) I mean the level of precision and detail at which a situation is characterized (how coarse-grained or fine-grained). This can be exemplified by an expression hierarchy like that in (6). Under appropriate circumstances, the same entity might be designated by any of these expressions.

( 6 ) thing $\rightarrow$ object $\rightarrow$ vehicle $\rightarrow$ truck $\rightarrow$ pick-up truck $\rightarrow$ battered old pick-up truck

Perspective is multifaceted. Two of its facets are vantage point, illustrated by the contrast in (7), and direction of mental scanning, exemplified in (8). Sentence (7a) construes the situation as being seen from a vantage point in the attic, (7b) from a vantage point down below. The sentences in (8) describe precisely the same situation. They contrast semantically by inducing us to mentally scan through the scene in opposite directions in building up to its full conception.

(7) a. Come on up into the attic!

b. Go on up into the attic!

(8) a. From home plate to the pitcher's mound, the grass has all been worn away.

b. From the pitcher's mound to home plate, the grass has all been worn away.

There are many kinds of prominence that need to be distinguished. Only two concern us directly, namely profiling and trajector/landmark organization. Each is strongly motivated in purely semantic terms, and subsequently proves essential for describing grammar (cf. Langacker (1993b, 1999c)).

Every expression evokes some conception - simple or complex - as the basis for its meaning. Within its conceptual base, an expression singles out a particular substructure as a kind of focus of attention. This substructure, called the profile, is the one the expression designates (its conceptual referent). For example, as sketched in (9a), the word arc evokes as its base the conception of a circle, within which it profiles any segment. (Observe that heavy lines indicate profiling.) The base for roof is the conception of a house, within which it profiles the upper part that covers it. Two expressions can have exactly the same base yet differ in meaning because of the alternate profiles they 
impose on it. For instance, husband and wife both evoke as their base the conception of a male (M) and a female (F) linked in a relationship of marriage (represented by double lines). The semantic contrast between them is not a matter of conceptual content, but rather one of prominence, the choice of profile.

(9)

b. roof

c. husband

d. wife
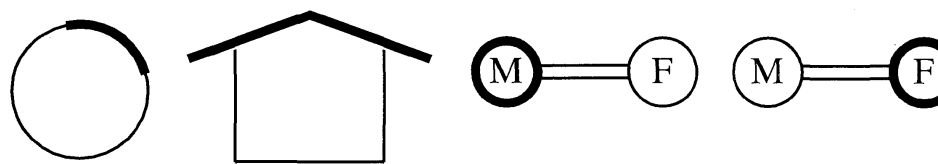

Crucially, an expression can profile either a thing or a relationship. Both notions are defined quite abstractly (Langacker (1987b)). Here I can merely note that things are not limited to objects or physical entities, and a relationship does not necessarily involve multiple participants. The expressions in (9) profile things. Some examples of profiled relationships are given in (10). As abbreviatory notations, I often employ circles or ellipses to represent things, and various kinds of lines or arrows for relationships. Note further that, because the conception of a relationship presupposes and incorporates the conception of its core participants, those participants are part of the profiled relation and are thus depicted with heavy lines.

a. smart

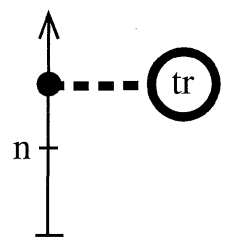

b. in

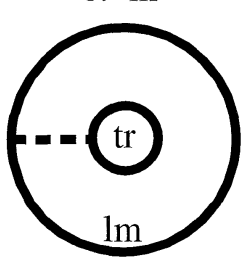

c. approach

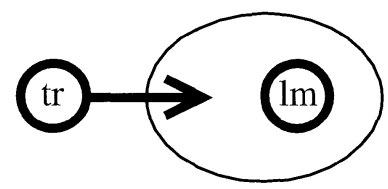

d. throw

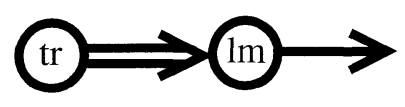

The adjective smart exemplifies a one-participant relation. The profiled relationship consists of this participant (shown as a circle, since a person is a kind of thing) being situated beyond the norm (n) on a 
scale of intelligence. Prototypically, the preposition in profiles a twoparticipant relationship of spatial inclusion (but cf. Vandeloise (1991: ch. 13)). The verb approach profiles an event in which one participant moves (single arrow) toward the other without reaching it, but does arrive in its neighborhood (given as an ellipse). In the case of throw, one participant exerts force (double arrow) on the other, causing it to move rapidly along an extended trajectory.

With expressions that profile relationships, a second kind of prominence comes into play. It consists in the degree of prominence conferred on the participants in the profiled relation. There is generally a primary focal participant, called the trajector (tr). This is the participant the expression is concerned with locating or characterizing. Often there is also a secondary focal participant, called a landmark $(\mathrm{lm})$. Metaphorically, we can think in terms of primary and secondary spotlights, which can be directed at different elements within the scene onstage. Trajector and landmark can also be characterized as primary and secondary figures within the profiled relationship (Langacker (1999c, 2001)).

Relational expressions that evoke essentially the same content for their base can nonetheless differ in meaning by virtue of their profiles and/or their trajector/landmark alignment. A well-known example is the contrast between like and please. For both, we can posit a conceptual base involving two participants, with the roles of stimulus and experiencer, which interact as shown in (11). The stimulus somehow impinges on the experiencer, who perceives or apprehends it and has a positive $(+)$ affective reaction. The verb like describes the experiencer's role in this interaction, so the experiencer functions as trajector, whereas please focuses the stimulus. Focusing one or the other participant naturally serves to highlight those aspects of the overall relationship it is responsible for. Consequently, the profile of like saliently includes the experiencer's apprehension of the stimulus, while that of please centers on the latter's stimulation of the former.

(11) a. like

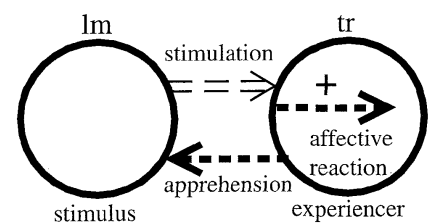

b. please

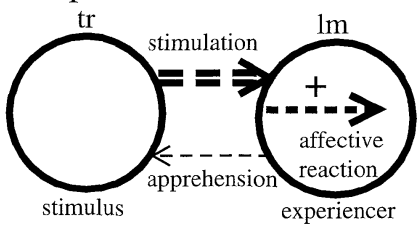

The constructs profile, trajector, and landmark are justified for pur- 
poses of semantic description but also prove essential to grammar. An expression's profile - not its overall conceptual content - is what determines its grammatical category. For instance, a noun profiles a thing, as in (9). Such classes as verbs, adjectives, adverbs, and prepositions profile various sorts of relationships. A verb designates a process, defined as a relationship followed in its evolution through time. The other classes mentioned profile relationships that are non-processual (or atemporal) - though time may well be involved, the profiled relationship is viewed holistically (rather than being scanned sequentially through time). They are distinguished by the nature of their focal participants. An adjective (e.g. smart) has a thing as trajector, but no focused landmark. An adverb is comparable except that its trajector is a relationship rather than a thing. By contrast, a preposition (e.g. in) does have a thing as focused landmark, while its trajector can either be a thing or a relationship.

Trajector/landmark organization provides the conceptual basis for the grammatical notions subject and object. A subject can be characterized as a nominal expression that specifies the trajector of a profiled relationship, and an object as one that specifies the landmark of a profiled relationship. Hence the subject of like, for example, designates the experiencer, and that of please the stimulus. Conversely for their objects.

\section{Prototypical Constructions}

A construction is simply an assembly of symbolic structures. The CG characterization is basically the same whether a construction is specific or schematic, whether it is fixed or novel, and whether it is morphological or syntactic.

In a typical construction, two component symbolic structures are integrated to form a composite symbolic structure. They are integrated at both the semantic and the phonological poles, their phonological integration serving to symbolize their semantic integration. At either pole, integration is effected by correspondences (marked by dotted lines) that equate particular elements within the two component structures. To form the composite structure, corresponding elements are superimposed, their specifications being merged (or unified). As a consequence, component elements that correspond each correspond to the merged composite element derived by their superimposition. 
Consider the phrase smart woman, sketched in (12). The two component structures, smart and woman, are shown at the bottom. The composite expression smart woman is shown at the top. At the semantic pole, the adjective smart profiles a relationship that situates its trajector on a scale of intelligence. The noun woman profiles a thing. To simplify the representation, its many semantic specifications are simply abbreviated as $W$. The semantic integration of smart and woman hinges on a correspondence between the adjective's trajector and the noun's profile. By superimposing these elements and merging their specifications, we obtain the composite semantic structure, in which a thing characterized as a woman is located on a scale of intelligence. The composite expression profiles the woman (a kind of thing), so the overall expression is classed as a noun.

(12)

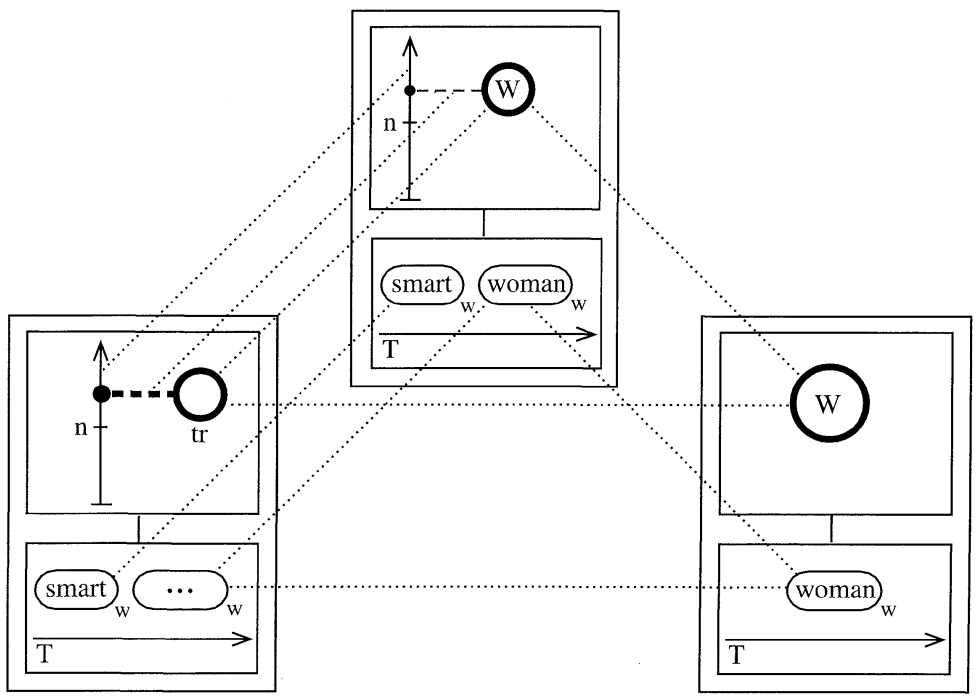

This semantic association of smart and woman is symbolized by the integration of these words at the phonological pole. (Words are indicated by ellipses subscripted with lower-case $w$.) That is, the fact that smart qualifies woman semantically is symbolized by the fact that these words occur together in the speech stream in a particular linear (i.e. temporal) order. The speech stream is represented diagrammatically by the arrow labeled $T$ (for processing time). The horizontal correspondence line equates woman with the word that directly follows smart in the speech stream. Phonologically, then, the composite expression derived by superimposing corresponding elements is smart woman. 
The dynamic language employed above-saying that the component structures are integrated to form the composite structure by superimposing and merging corresponding elements - should not be taken too seriously. It is not being claimed that, in terms of actual processing, the component structures exist first, and the composite structure only subsequently. Nor is the composite structure seen as being constructed out of the component structures, which supply all its content. The composite structure is viewed as an entity in its own right, which may have properties not derived from either component.

More neutrally, then, I say that a construction is an assembly of symbolic structures linked by correspondences and categorizing relationships. Diagram (12) illustrates how they are linked by horizontal and vertical correspondences. I will now describe how they are also linked by categorizing relationships. Though I will concentrate on the semantic pole, all the constructions discussed must be understood as being bipolar.

It is typical for one component structure to contain a salient schematic element which the other component structure serves to elaborate. This schematic element, corresponding to the profile of the other component, is called an elaboration site (or e-site) and is marked by shading. In (13), the semantic pole of smart woman, the elaboration site is the trajector of smart. The trajector is quite salient, the primary focus within the profiled relationship. Within the adjective itself it is also quite schematic; elaboration by woman serves to make it more specific.

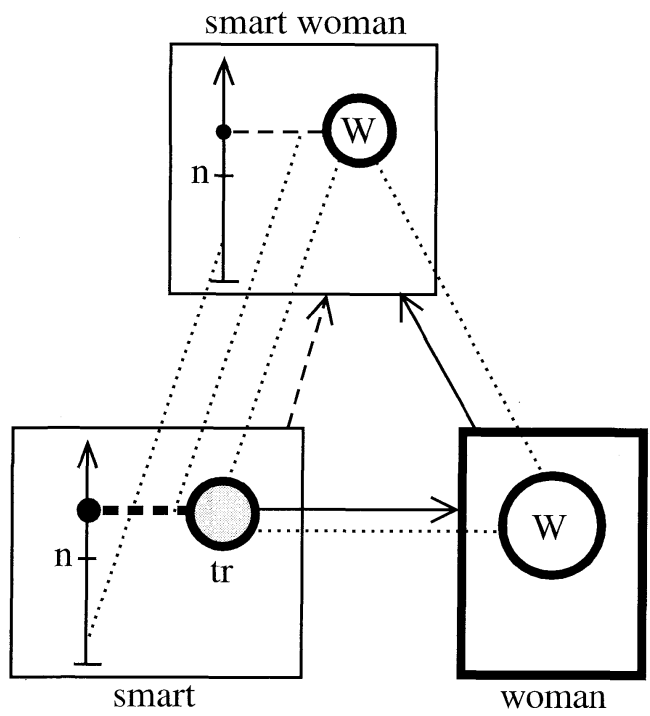


The vertical arrows in (13) indicate that the two component structures (taken as wholes) categorize the composite structure (taken as a whole). In what sense is their relationship one of categorization? Within a construction, the composite structure has special status: it stands in the foreground as the structure primarily employed for higher level purposes. The component structures tend not to be invoked for their own sake, but rather as stepping stones allowing one to arrive at the composite structure. I take this asymmetry as being a special case of the asymmetry inherent in the relation between a categorizing structure and the target of categorization. Moreover, the composite structure is an entity in its own right, often with special properties not strictly derivable from the meanings of component elements considered individually. In other words, the composite structure is not literally constructed out of the components - the stepping stones are not building blocks. Rather, the components serve merely to evoke and motivate certain facets of the composite conception. As a general matter, the relation between them is more akin to categorization than strict composition.

In (13), the categorizing relationship between the component structure woman and the composite structure smart woman is one of elaboration (solid arrow). This is because the two are fully consistent in their specifications and smart woman offers a finer-grained characterization of the profiled entity. On the other hand, the relation between smart and smart woman is given with a dashed arrow, indicating extension rather than elaboration. Considered as wholes, smart and smart woman are inconsistent in their specifications, particularly in regard to profiling: smart profiles a non-processual relationship, whereas smart woman profiles a thing. Thus, while smart contributes to the composite conception (or motivates a certain aspect of it), it is not precisely schematic with respect to it.

This is quite typical. In a construction, it is normally the case that the profile of one component structure, but not of the other, corresponds to the composite structure profile. The component structure whose profile is thus inherited at the composite structure level is called the profile determinant. Diagrammatically, the profile determinant is enclosed in a heavy-line box. In (13), woman functions as profile determinant because smart woman designates the woman, not the relationship of being intelligent.

The phrase smart woman represents a specific symbolic assembly, i.e. 
an expression. This expression instantiates a constructional schema describing a general syntactic pattern for combining adjectives with nouns. Diagrammed in (14) is the semantic pole of this schema, representing the abstracted commonality of countless adjective+noun sequences. The component structure on the left is the schematic representation of an adjective: it profiles a non-processual relationship of unspecified nature, except that its trajector is a thing, with no focused landmark. The component structure on the right is the schematic representation of a noun, which profiles a thing. The adjectival trajector functions as elaboration site and corresponds to the nominal profile. The noun is the profile determinant, so the composite structure profiles a thing which, as an unprofiled part of its conceptual base, participates in the relationship coded by the adjective. At the phonological pole, the schema specifies that the adjective directly precedes the noun in the speech stream.

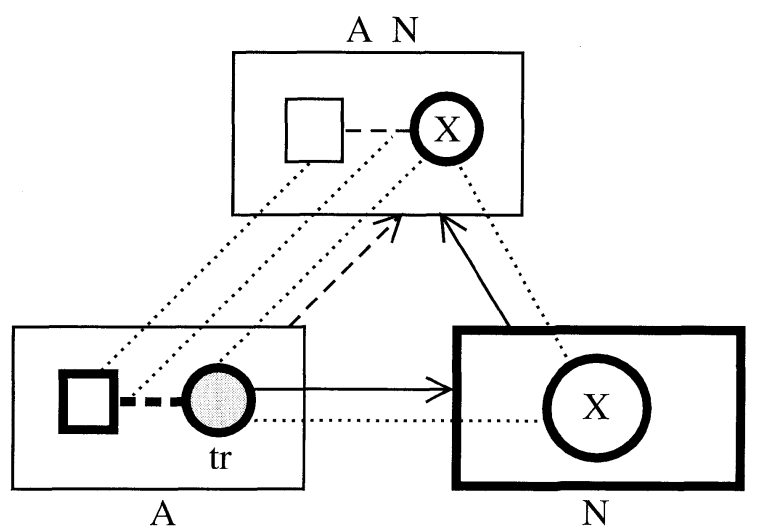

Constructional schemas provide the patterns a language makes available for the production of complex expressions. These schemas are themselves symbolic assemblies, hence meaningful, although their meanings are generally quite abstract. Their skeletal meanings are immanent in (i.e. they lie within) those of instantiating expressions, which elaborate them (flesh them out) in their own individual ways.

A constructional schema's semantic pole constitutes a constructional meaning, the schema's contribution to the overall meaning of composite expressions. With more abstract schemas, like (14), constructional meaning is limited to specifying the grammatical category of symbolic elements, as well as organizational properties: how these elements relate to one another in terms of correspondences, categorization, and 
profile determinance. For instance, the specification that smart woman designates the woman (rather than the property of being intelligent) is a function of the entire construction, not of the component lexical items. It is likewise an aspect of constructional meaning that the profiled woman is the trajector of smart (the person whose intelligence is specified), rather than having some other role.

Whether specific or schematic, symbolic assemblies can in principle be of any size. When there are more than two component structures, it is usual for an assembly to exhibit multiple levels of organization, such that a composite structure at one level functions in turn as component structure with respect to another, higher level. The result is a kind of constituency. However, the constituency hierarchies posited in CG are not comparable to the syntactic tree structures of generative grammar, which are generally conceived as purely formal objects with no intrinsic conceptual or phonological content. On the contrary, CG constituency hierarchies consist solely of symbolic structures, each comprising a semantic and a phonological pole. Grammatical constituency is simply the order in which simpler symbolic structures are progressively integrated to form more complex ones.

Consider the nominal expression smart woman with a PhD, sketched in (15) (ignoring the article). It consists of several canonical constructions: smart woman, already examined; a prepositional phrase, where with takes a nominal object; and the higher level construction where the composite expressions smart woman and with a PhD combine as component structures to form the overall expression. 
(15)

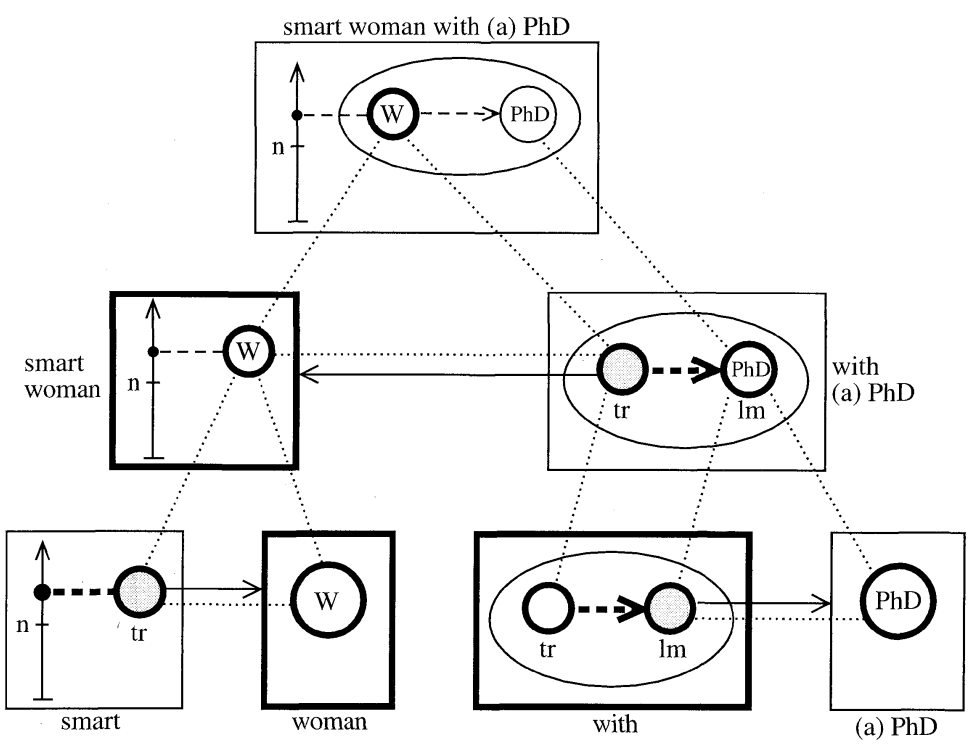

The representation of with is meant to indicate that it profiles a nonprocessual, essentially possessive relationship such that the trajector anchors an experiential dominion (Langacker (1993c)) in which the landmark can be found. The schematic landmark functions as e-site, corresponding to the profile of the nominal component $a \mathrm{PhD}$. Since the composite structure with a PhD profiles the possessive relationship (not the academic degree), with is the profile determinant at this level. At the higher level, the schematic trajector of the prepositional phrase corresponds to the profile of smart woman, which elaborates it and imposes its own profile on the higher level composite structure. Hence the overall expression, smart woman with a PhD, designates the woman.

Some basic grammatical notions are straightforwardly characterized in terms of symbolic assemblies as described thus far. As the term is most commonly understood, for example, a head can be defined as the profile determinant at a given level of organization (cf. Zwicky (1985), Hudson (1987)). It is the component structure whose profile is inherited at the composite structure level, thereby determining the grammatical category of the composite expression. Woman is thus the head in the nominal expression smart woman, and with in the prepositional phrase with a PhD. Granted the constituency shown in (15), the head at the higher level of organization, for the expression as a whole, is 
smart woman (and by extension, woman-as the head within the head).

We can go on to characterize the notions complement and modifier in terms of whether a component structure elaborates or is elaborated by the head. More specifically, a complement is a component structure which elaborates a salient substructure of the head. In (15), the nominal expression $a P h D$ is thus a complement of with, since it elaborates a salient substructure of with, namely its landmark (a focal participant). Conversely, a modifier is a component structure a salient substructure of which is elaborated by the head. Hence smart modifies woman in (15), since the head-woman-elaborates its trajector. In the same way, with a PhD modifies smart woman at the higher level.

I should emphasize that these definitions refer exclusively to conceptual factors-profiling, profile determinance, correspondence, elaboration-observable at the semantic pole of symbolic assemblies. Despite their utility for describing grammar, these constructs are ultimately semantic in nature, not autonomous grammatical primitives.

\section{Non-prototypical Constructions}

The constructions examined so far are reasonably considered canonical, or prototypical. They have a number of typical properties: (i) there are two component structures; (ii) one component profiles a thing, the other a relationship; (iii) the nominal profile corresponds to a focal participant of the relationship (its trajector or landmark); (iv) that participant is schematic, being elaborated by the nominal component; (v) the composite structure inherits its profile from one of the two component structures.

Grammatical constructions are nonetheless highly varied and deviate from the prototype in myriad ways (Langacker (1988b, 1999b, To appear b)). Ultimately, it is only required that a construction comprise an assembly of symbolic structures linked by correspondences. Even this must be qualified if we make the terminological decision to regard single morphemes as constructions, so that all of lexicon and grammar can be described as residing in constructions. With this approach, a morpheme constitutes a degenerate construction, a symbolic assembly consisting of just one symbolic element. Hence there is no distinction between component and composite structures, nor any correspondences. 
Also deviating from the prototype are constructions with more than two component structures. The previous example, smart woman with a PhD, might be analyzed in this fashion. On this account, diagrammed in (16), smart and with a PhD modify woman at the same level of constituency, in a tripartite construction (I omit the internal structure of the prepositional phrase). It will be observed that, despite the difference in constituency, the overall composite structures in (15) and (16) are identical, and the necessary semantic and grammatical relationships are expressed in both (e.g. woman is the head, modified by smart and with a PhD).

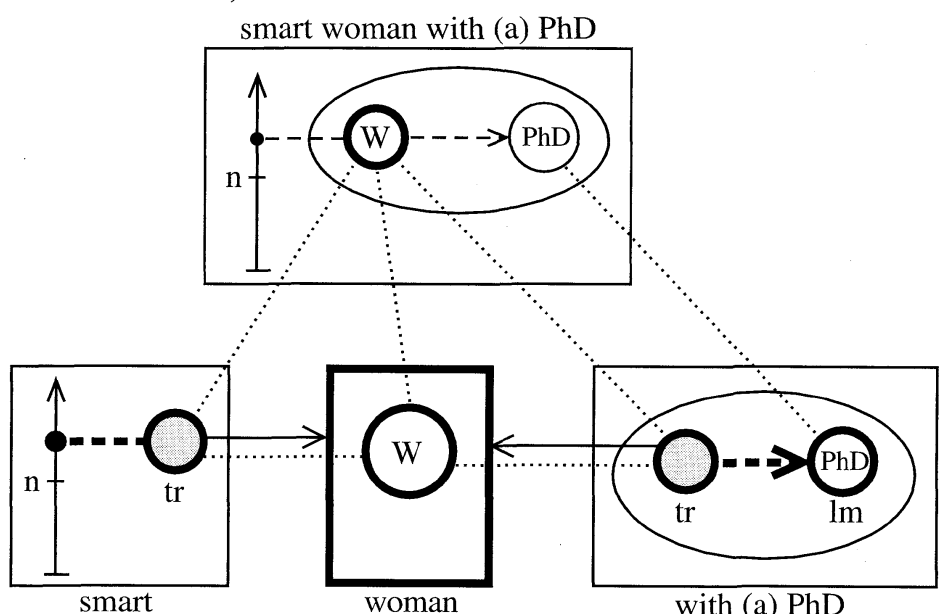

Which constituency is correct, the one in (15) or the one in (16)? Actually, I suspect both of them are. In CG, essential grammatical relationships are conceptual in nature and captured by correspondences, not in terms of constituent structure. The same relationships can be captured with alternate constituencies, with the empirical consequence that constituent structure is often flexible, variable, and even indeterminate (Langacker $(1995,1997)$ ). In the case at hand, evidence for two alternate constituencies is provided by the intonational possibilities in (17), where a slash (/) indicates a slight pause. It is further corroborated by the ability of one to refer anaphorically to either smart woman or just woman, as seen in (18).

(17) a. smart woman/with a $\mathrm{PhD}$

b. smart/woman/with a $\mathrm{PhD}$

(18) a. They're looking for a smart woman with a $\mathrm{PhD}$, not one with just a masters. 
b. A smart woman with a $\mathrm{PhD}$ is happier than a brilliant one with just a masters.

More generally, a number of adjectival modifiers can be strung together with no indication of any particular constituency hierarchy, especially when pronounced with pauses between them and with equal degrees of stress. In this case I see no reason not to posit a multipartite construction, as seen in (19). Each adjective ascribes a property to the modified noun, so the trajector of each corresponds to its profile. (Here I simplify by not indicating elaboration or e-sites.)

(19) big / ugly / vicious / dog

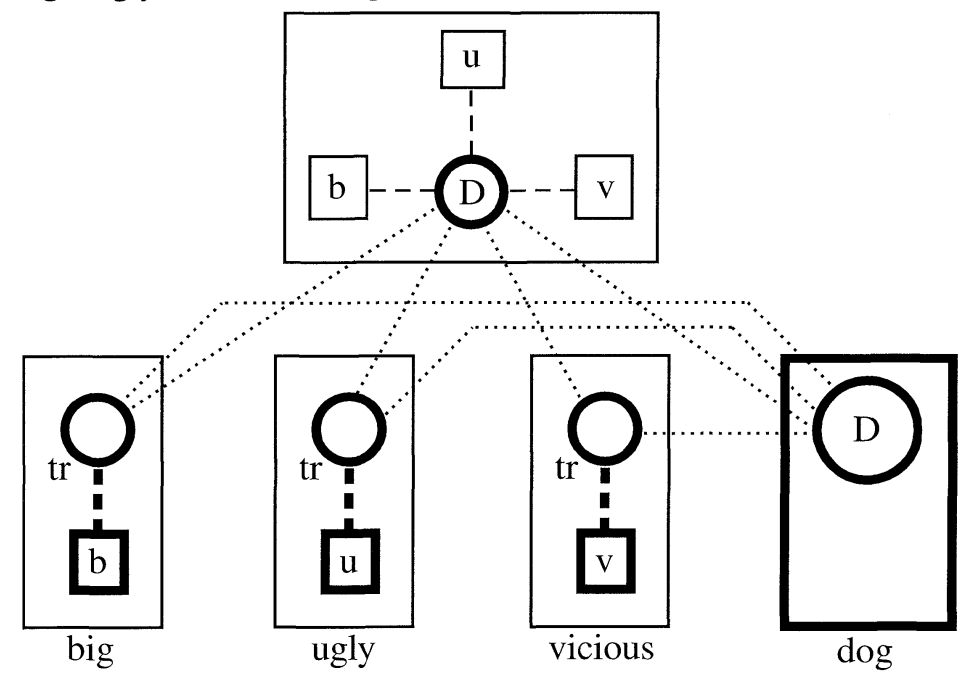

Many constructions depart from the prototype because they lack a head, or profile determinant, defined as a single component structure whose profile corresponds to the composite structure profile. Here we can distinguish three subcases. A unique profile determinant may be absent (i) because the component structure profiles correspond to one another, so they all correspond to the composite structure profile; (ii) because the composite structure profile represents a conflation of the component structure profiles and is not equivalent to any one of them individually; or (iii) because the composite structure profile is distinct from that of any component.

The first case is exemplified by appositional constructions, where two nominal expressions each describe the same nominal referent, in different ways. The nominal components range in size from simple nouns 
to full noun phrases:

(20) a. pussy cat; sailor boy

b. my friend Henry Kissinger; the famous French novelist Marcel Proust

c. the $\{$ fact/claim/idea/notion/myth $\}$ that syntax is autonomous

Abstractly, such expressions have the organization sketched in (21). Each component structure profiles a thing, these things correspond, and both correspond to the composite structure profile.

(21)

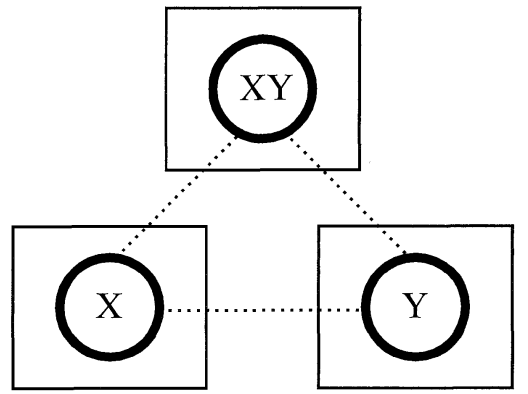

In cases like (20), we could make either of two terminological decisions: to say that both component structures are heads, or that neither is (since neither functions uniquely as profile determinant). I tend to follow the latter practice, essentially arbitrarily. I make the same terminological choice in the situation where the composite structure profile conflates the profiles of its components, none of which is thus equivalent to it taken individually. A favorite example is the nested locative construction:

(22) The hammer is in the garage, on the workbench, behind the electric saw.

Any number of locatives can be strung together in this manner, with no apparent grouping into constituents. Each successive locative specifies the trajector's location with greater precision (confines it to a smaller area). For our purposes, the important point is that the composite locative expression - in the garage, on the workbench, behind the electric saw-simultaneously locates the trajector with respect to three different landmarks. No one of these locative relationships stands out as the single location described by the overall expression. Rather, as shown in (23), all three specifications are simultaneously valid and equally focused. The profiled relationship is complex, for it evidently conflates the simple relationships expressed by the individual compo- 
nent structures.

(23)

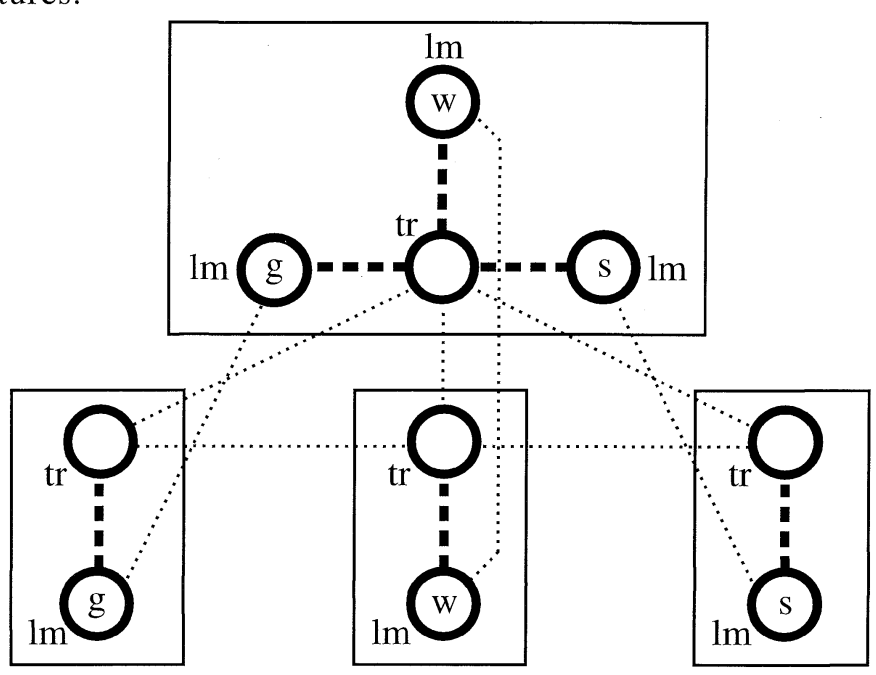

In the third type of construction lacking a head (traditionally called exocentric) the composite structure profile is distinct from that of both component structures. An example is pickpocket. In the verbal expression pick someone's pocket, the verb pick has the conceptual value sketched at the lower left in (24): the trajector exerts a force (double arrow), thereby inducing some object to move (single arrow). This object moves from its original location, which is focused as the landmark, into the trajector's dominion (sphere of control). The noun pocket designates a location, shown as a rectangle, which functions as a kind of container. The circle within it represents the contents of the container, while the larger circle represents the article of clothing of which it is a part. In the compound pickpocket, correspondences identify the contents of the pocket with the object that moves, and the pocket itself with the landmark of pick (the location emptied of its contents). However, the composite structure does not inherit the profile of either pick (the action) or pocket (the location). Instead it profiles the actor, corresponding to pick's trajector. Thus neither element of the compound functions as profile determinant. 
(24)

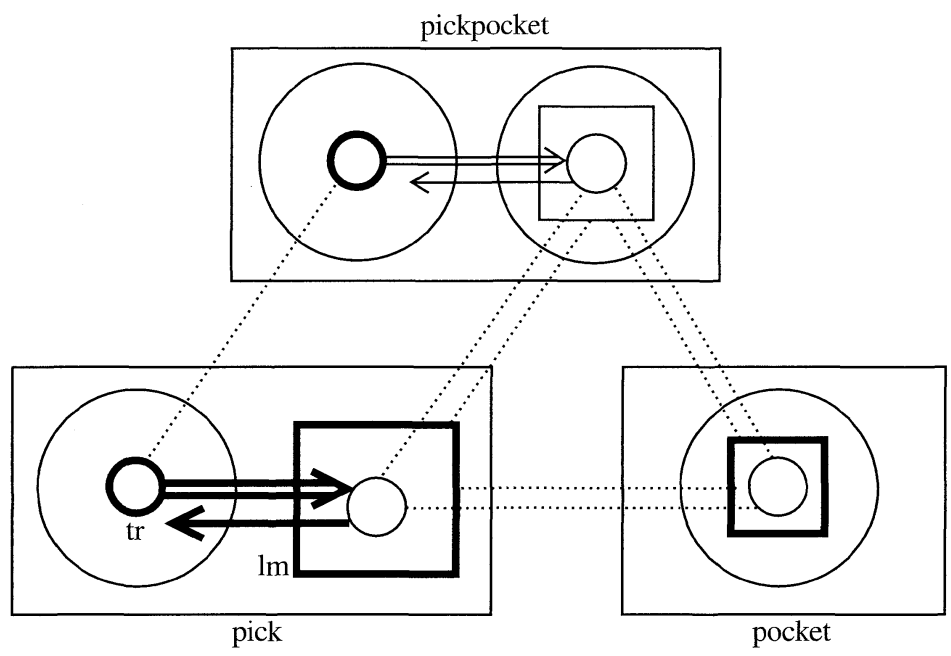

Pickpocket is idiosyncratic, in that the second element of an English compound normally functions as profile determinant. This can be contrasted with cases where an aspect of constructional meaning, while not inherited from either component, is nonetheless regular in the sense that it is specified by a productive constructional schema. Consider equative sentences in those languages where referential identity is marked simply by juxtaposing two nominal expressions. In Luiseño (a Native American language), a sentence like (25) predicates identity despite the absence of any verb or morphological element expressing this meaning.

(25) Wunaal ya'ash no-kaytu. (that man my-enemy)

'That man is my enemy.'

This is not an idiosyncratic expression but a regular construction, where equative sentences are productively formed using any appropriate combination of nominal expressions (NML). The constructional schema specifies that both component structures profile things, whereas the composite structure profiles a relationship of identity between them (given as a double line). The relationship profiled by the clause emerges at the level of the overall construction rather than coming from either component, but does so in accordance with a productive pattern. A particular expression like (26) is thus quite regular in formation, despite the absence of a head. 
(26)

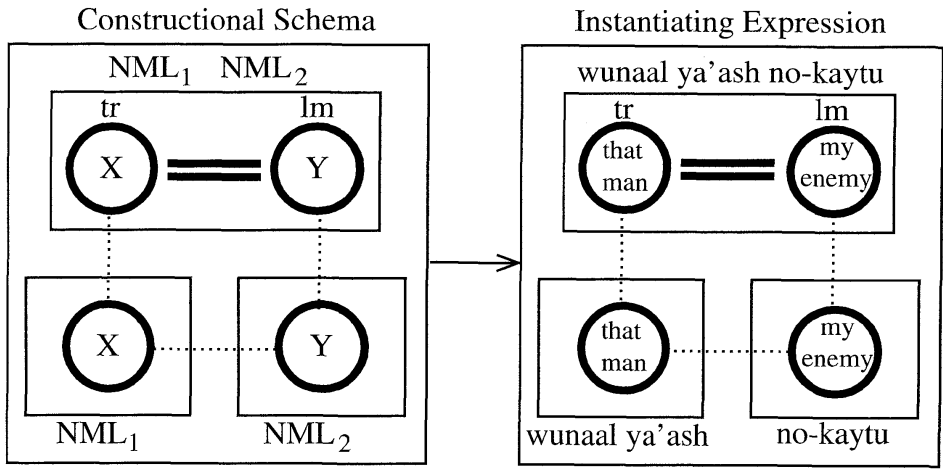

Equational and appositional constructions are non-prototypical in that both component structures profile things. Grammatical combination does not require a predicate-argument relationship, such that a nominal component specifies a relational participant. Moreover, an elaboration site does not have to be a thing, but may itself be a relationship. This is the case with adverbs, e.g. fast, whose trajector is a process situated on a scale of rapidity. In (27), a box represents the schematic process functioning as the adverb's trajector. It will be seen that a phrase like move fast is analogous to smart woman.

(27)

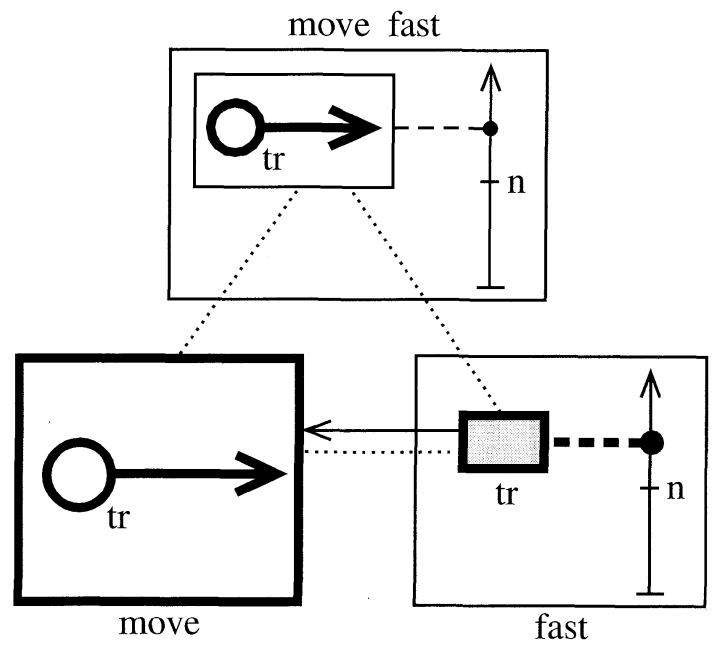

Nor is it required that an elaboration site be a focal participant. An e-site, defined as a substructure corresponding to the profile of the other component, need not even be particularly salient within the elaborated structure. An example is the compound woman smart, 
which is made-up but perfectly natural and well-formed. I interpret it as meaning 'smart in regard to women.' We know that people often exhibit intelligence with respect to certain topics but not others. The notation in (28) is meant to indicate that the property of being smart holds only in a particular domain of knowledge (represented as an ellipse), namely the one centered on a particular topic (given as a circle). This topic functions as e-site, being elaborated by woman. Though pivotal to the expression's interpretation, this e-site is not a focal participant of smart, nor is it highly salient.

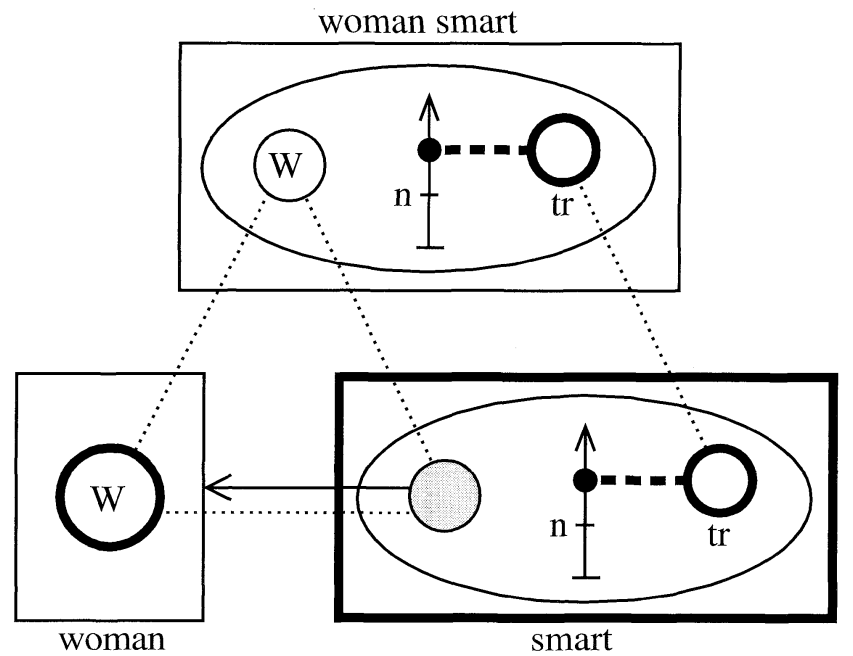

Observe that the phrase smart woman and the compound woman smart have the same component structures but very different composite meanings. They differ in their constructional meanings, contributed by the constructional schemas they instantiate. Smart woman instantiates the schema for the adjectival modification of nouns, sketched in (14). On the other hand, woman smart instantiates a semantically more flexible schema for compounds, where in general the second element functions as profile determinant. Thus, whereas smart woman profiles the woman, the compound woman smart profiles the relationship.

Of course, it is not even necessary that there be an e-site at all. Two component structures are capable of combining grammatically even in cases where neither contains a substructure corresponding to the other's profile. Consider the composite expression go away angry (e.g. Don't go away angry!). The meaning of go away makes no intrinsic reference to the mental state of its trajector, nor does angry 
evoke an action which this mental state accompanies.

Their integration is sketched in (29). The arrow labeled $t$ stands for time. As a complex verb, go away profiles a process, where development through time is salient as a matter of definition. The solid bar along the time arrow represents the span of time through which the event is followed in its temporal evolution. Being an adjective, angry merely profiles the situation of its trajector exhibiting a certain property. Continuation through time is not essential to its characterizationif a person is angry during a certain span of time, that person is angry at any single instant during that time span. It is however part of our understanding of angry that this emotion occurs in bounded episodes, enduring for some time on each occasion. A bar along the time arrow represents the duration of one such episode.

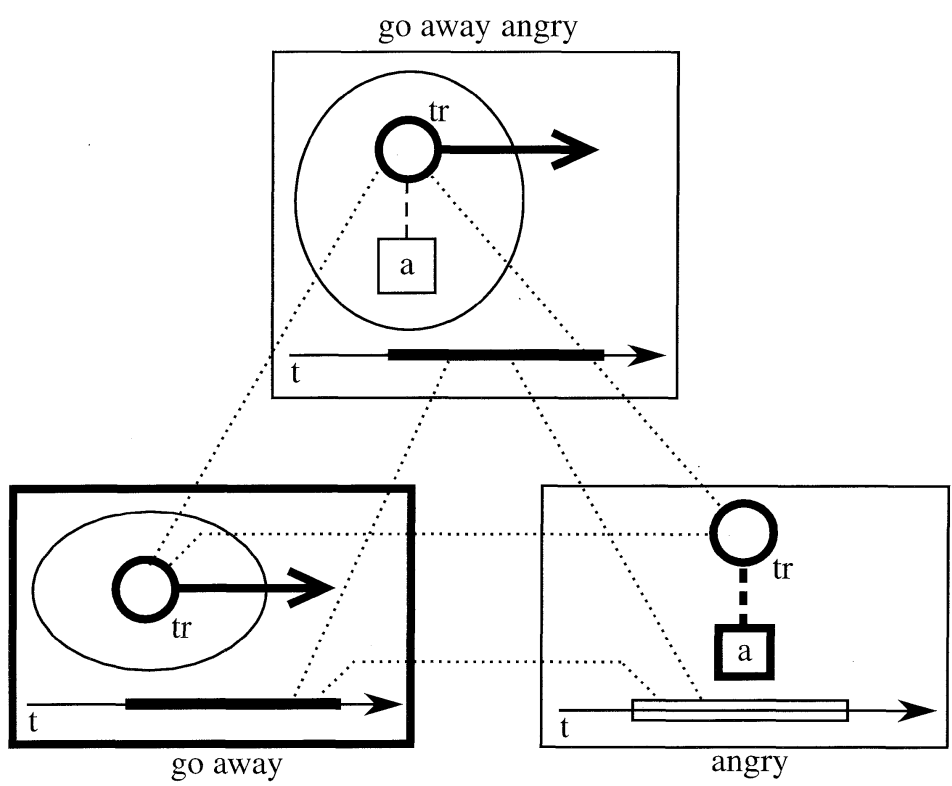

Although neither component structure elaborates a salient substructure of the other, they are integrated by virtue of two correspondences. First, their trajectors correspond - the person who goes away is the one who is angry. Second, the span of time through which the departure occurs is equated with the time span constituting one episode of anger; the expression cannot mean that the trajector goes away at one time and is angry at another, only that the two are temporally coincident.

Because neither component structure elaborates a salient e-site with- 
in the other, we cannot describe angry as either a complement or a modifier of go away. In cases like this the non-head component (angry) is generally called an adjunct. I should note that in CG the status of elements as complements, modifiers, or adjuncts is a matter of degree, reflecting the relative salience of particular notions within the global meanings of component structures. It is neither expected nor required that a particular term be obviously or uniquely applicable. That is, notions like complement, modifier, and adjunct are not unanalyzable grammatical primitives, but rather convenient labels for typical sorts of configurations that emerge with various degrees of distinctness in grammatical constructions.

An e-site sometimes exhausts the content of a component structure, rather than being limited to a proper substructure of it. This is commonly the case with derivational elements, which I generally analyze as being schematic for the category they derive. Consider the nominalizing suffix -er, as in swimmer, complainer, teacher, philanderer, etc. Prototypically, it forms a noun designating some kind of actor. As shown in (30), it can then be characterized as evoking for its base the schematic conception of an active process, which I have indicated by means of an arrow with ellipses (...). Within this base, it profiles the actor, a thing. The schematic process, representing the entire conceptual content of the suffix, functions as e-site in this construction, being elaborated by a specific verb, in this case throw. Since -er is the profile determinant, the composite expression designates the actor in the specific process of throwing, and since it profiles a thing, thrower is a noun.

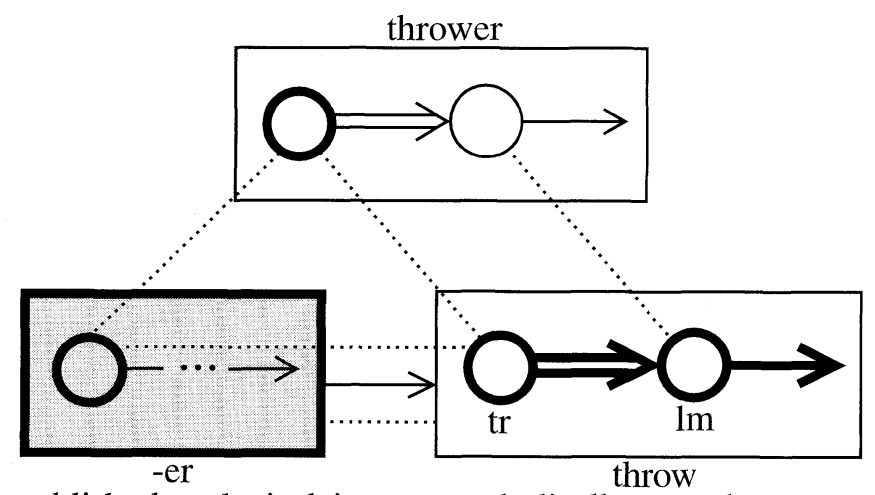

When established as lexical items, symbolically complex expressions vary in their degree of analyzability, defined as the extent to which speakers recognize the semantic contributions of component elements. 
Novel expressions are fully analyzable, since the speaker has to construct them from component elements on the basis of their meanings. Established expressions may be less analyzable. They come as prepackaged assemblies, whose composite forms and meanings are wellknown and well-rehearsed, so it is not essential that the component structures be mentally accessed individually. In fixed and frequently occurring expressions, there is thus an overall tendency for component elements to be activated only to a lesser degree, and perhaps not on every occasion of their use.

The result is that familiar expressions can often be ranked in terms of their degree of analyzability, e.g. flinger $>$ complainer $>$ computer $>$ propeller $>$ drawer. A novel expression like flinger 'something that flings' is fully analyzable. The lexical item complainer is highly analyzable (it is always understood as 'one who complains'), but the others listed are progressively less so. In using the term computer, we do not always specifically think of it as 'something that computes,' and a propeller is seldom thought of as 'something that propels.' At the extreme endpoint of the scale, a form like drawer may be fully unanalyzable, in which case it constitutes a single morpheme.

Degree of analyzability is an important dimension of linguistic organization which has largely been neglected. It is unproblematic in CG, where constructions are viewed as assemblies of symbolic structures. Since the composite structure is a distinct entity, existing in its own right, in established expressions it can perfectly well be activated independently of the component structures. The contrast between a fully analyzable expression like the novel flinger 'something that flings' and a partially analyzable form like computer is represented in (31). Words in capital letters are used here to abbreviate the semantic structures, and lower-case letters for phonological structures. Dashed-line boxes enclose structures that are activated only partially or only sporadically.

a. Fully Analyzable Expression

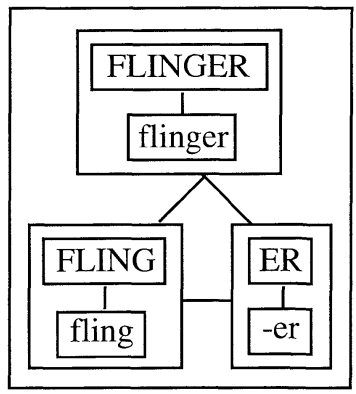

b. Partially Analyzable Expression

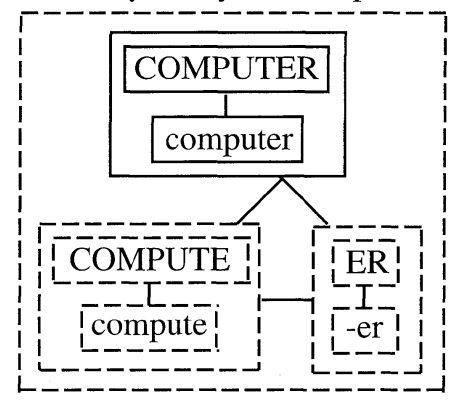


On this view, the analyzability of a composite expression into component morphemes is a matter of degree - a form like propeller is neither completely monomorphemic nor completely bimorphemic. Moreover, once degree of analyzability is recognized and accommodated, other well-known problems of classic morphemic analysis disappear (Langacker (1995)).

First, as seen in (32a), it is quite possible for just one symbolic component to be recognized within a more complex expression. For instance, the day of Monday, Tuesday, Wednesday, etc. is certainly recognized by contemporary speakers, but the residue (Mon, Tues, Wednes, etc.) is not. We can simply characterize these expressions as defective constructions having a composite structure but only one (partially recognized) component structure.

(32)

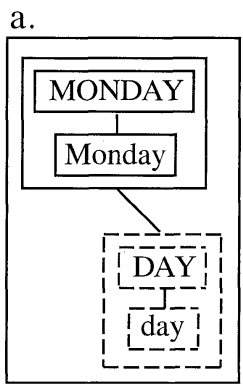

b.

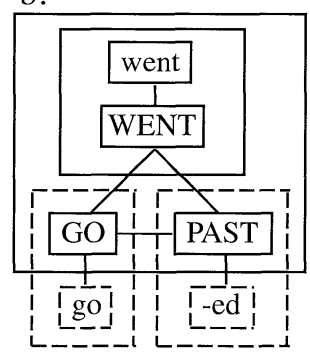

c.

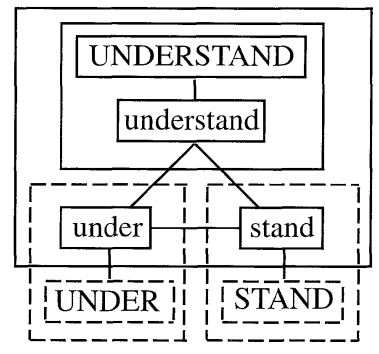

Beyond this, we can readily handle cases of phonological suppletion and semantic opacity. A case of suppletion is went, the past tense of go. Semantically the components GO and PAST are clearly evident, but phonologically there is just a single, essentially unanalyzable form. As shown in (32b), the symbolic unit went can be characterized as comprising a full, bipolar composite structure, while being defective in regard to component structures, which have a semantic pole but not a phonological pole.

Conversely, as shown in (32c), it is possible for a construction to be defective by including only the phonological poles of the component structures. An example is understand, which is clearly analyzable into the morphological elements under and stand, but which speakers find semantically opaque, making no connection to the meanings of the preposition and the verb. In the case of both went and understand the only symbolic link is at the composite structure level. However, these constructions still conform to the content requirement. 


\section{Grammatical Dependencies}

In CG, grammatical dependencies are non-configurational. They reside in semantic relationships, primarily correspondences between conceptual substructures, not in any particular constituency configuration. Two basic kinds of grammatical dependencies are subject and object relationships. Both subjects and objects are complements: nominal expressions which respectively elaborate the trajector and the landmark of a profiled relationship.

Let us take the expression Jennifer likes that boy. It is normally ascribed the constituency shown in (33a), where the verb and object form a constituent excluding the subject. This corresponds to the normal intonational grouping, where a slight pause is possible between Jennifer and likes that boy: Jennifer/likes that boy. However, in deliberate speech it is possible to divide the sentence into three intonational units: Jennifer/likes/that boy. This suggests a tripartite structure, and while I do not claim that intonation always correlates with constituency, it is at least one factor that bears on it. As a secondary pattern, I presume that English allows the constituency shown in (33b). The configurational difference between $(33 a, b)$ has no effect on grammatical dependencies. In both structures, by tracing horizontal and vertical correspondence lines we find that Jennifer functions as the subject of like, and that boy as its object.

(33) a.

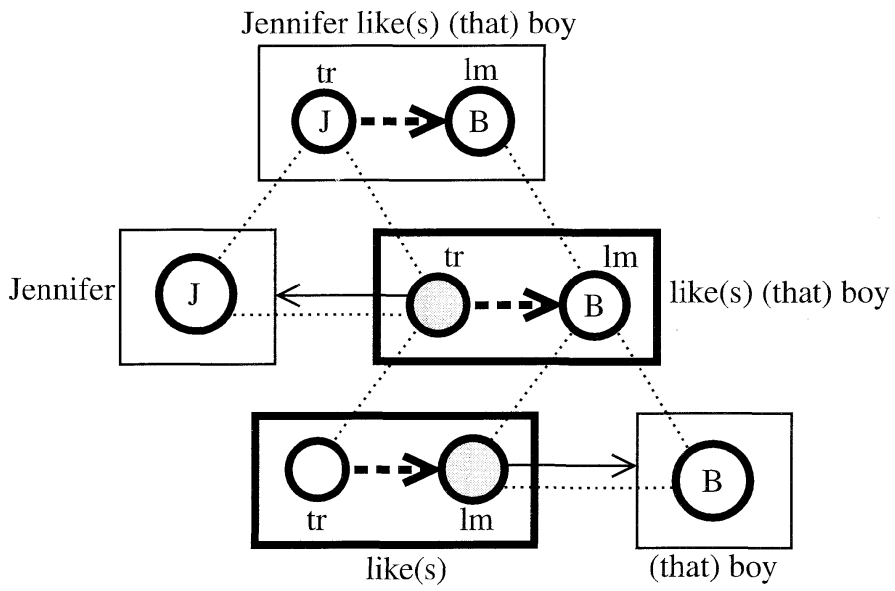


b.

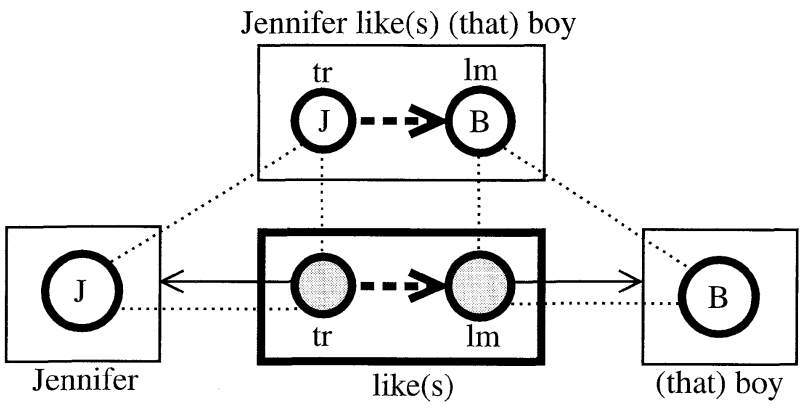

c.

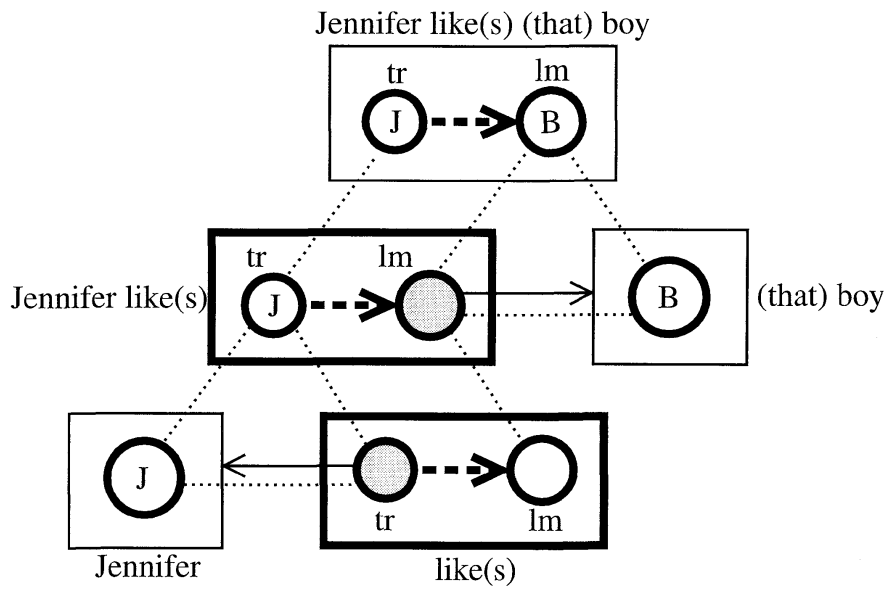

What about the other possibility, where subject and verb form a constituent, as shown in (33c)? This too gives rise to the same composite structure and the same grammatical relations: Jennifer as subject, that boy as object. Though a pause after like is not very natural (?Jennifer likes/that boy), there are various more elaborate constructions where positing a subject+verb constituent does seem necessary. Among these are a clause-internal topic construction, as in (34a), coordinate structures like (34b), and object relative clauses, as in $(34 \mathrm{c}, \mathrm{d})$. To accommodate such expressions, I would simply posit a constructional schema allowing subject and verb to combine directly. This schema may be limited to larger contexts, represented by more complex constructional schemas, in which the verb's landmark is elaborated at a higher level of grammatical organization. In (34a), for instance, the landmark of likes is specified in a higher level construction by a nominal functioning simultaneously as object and clausal topic. 
(34) a. That boy Jennifer likes (this one she doesn't).

b. Jennifer likes, and Sharon really admires, the boy who lives next door.

c. The boy that Jennifer likes finally called.

d. The boy finally called that Jennifer likes.

This approach to grammatical dependencies easily accommodates various phenomena that were handled in classic transformational grammar by means of derivations from underlying structures. An example is relative clause extraposition. Because sentences like $(34 \mathrm{c}, \mathrm{d})$ are effectively equivalent semantically, and modification was thought to require grammatical co-constituency, a deep structure analogous to $(34 \mathrm{c})$ was posited for both expressions. Sentences like (34d) were then derived by an optional transformation serving to extrapose the relative.

The CG account is sketched in (35), ignoring everything not essential to the point at hand. In (35a), the relative clause combines with the head noun in a canonical modifying construction. I have not shown the internal structure of the relative, which is a subject+verb constituent, equivalent to the lower portion of (33c). At the composite structure level, it profiles the process of liking, with a specified trajector (Jennifer) and a schematic landmark. It modifies the head noun, boy, by virtue of a correspondence between its landmark and the nominal profile. This correspondence identifies boy as the semantic object of like. At the higher level of organization, the subject nominal the boy that Jennifer likes elaborates the trajector of call (whose processual profile is simply represented as a solid arrow).

(35) a.

(the) boy (that) Jennifer like(s) (finally) call(ed)

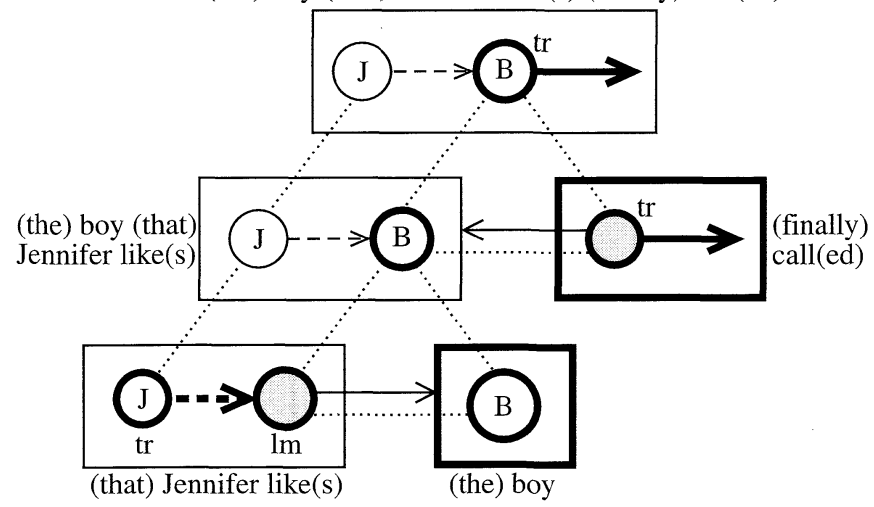


b.

(the) boy (finally) call(ed) (that) Jennifer like(s)

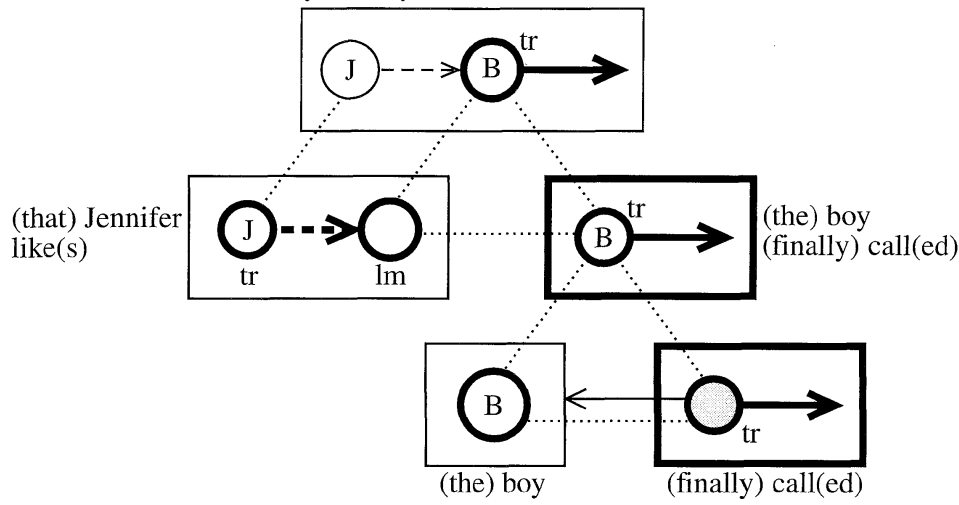

The alternative expression in (34d), with an extraposed relative, is diagrammed in (35b). Here the boy and finally called are integrated in the usual fashion to form a clause at the lower level of constituency. The relative, that Jennifer likes, combines with this clause as a whole in the higher level construction. However, while the two clauses combine as wholes, their integration hinges specifically on a correspondence between the landmark of the relative and the main clause trajector. Owing to this correspondence, the relative functions to describe the boy, which is identified as the semantic object of like. This is not a canonical modifying construction, since the head-which is the full main clause, the boy finally called-does not per se elaborate the landmark of the relative. The semantic effect of modification is nonetheless achieved.

Relative clause extraposition does not come for free-speakers have to learn the construction (and the various restrictions on it), and linguists have to describe it by positing the appropriate constructional schemas. Still, this alternative grammatical option does not require any special theoretical apparatus (like deep structures and transformations). It is straightforwardly accommodated with the same theoretical devices employed for the basic pattern.

In $(35 a, b)$, correspondences identify the boy as being both the trajector of call and the landmark of like. Accordingly, in the composite semantic structure this same individual is simultaneously depicted as participating in both relationships. It is usual and unproblematic for the same conceptual element to have multiple roles, each pertaining to a particular relationship coded at a particular level of organization. In 
(36), for example, a rock functions simultaneously as the landmark (object) of throw and as the trajector (subject) of into the pond.

(36) She threw a rock into the pond.

Their integration is sketched in (37). At the first level of constituency, a rock elaborates the landmark of throw in a normal direct object construction. Throw implies that the landmark, due to the force exerted by the trajector, moves rapidly along an extended spatial path. Throw itself of course evokes this path only schematically. Still, the conception of the landmark successively occupying all the spatial positions defining a path is an inherent aspect of throw's meaning, and in the higher level construction it functions as an elaboration site. Shown as a rectangle in (37), this substructure of throw a rock is elaborated by the path prepositional phrase into the pond, which describes the path in more specific detail. Through horizontal and vertical correspondences, the rock is thus identified as both the landmark of throw and the trajector of into the pond.

(37)

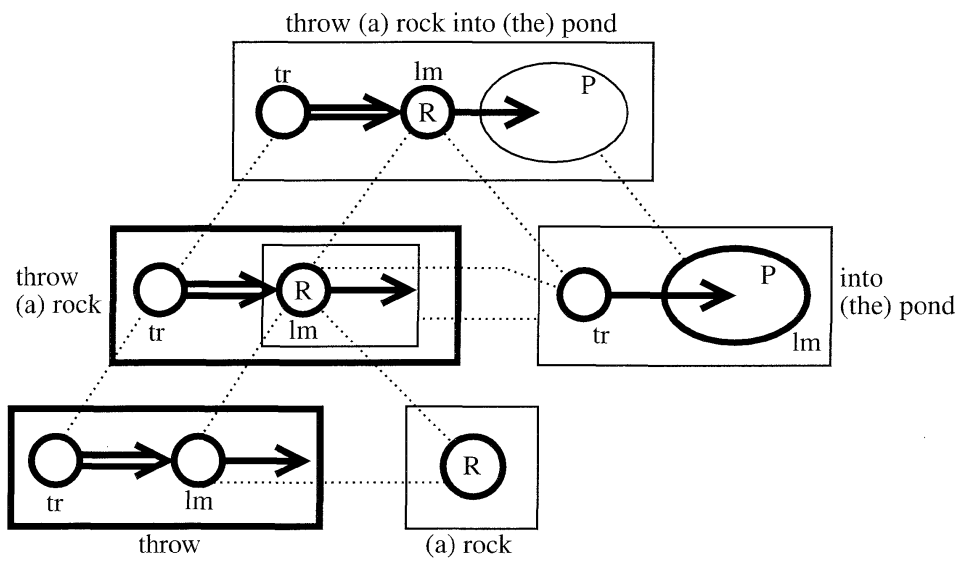

A final, more elaborate example is the following Luiseno sentence (cf. Langacker (1973)):

(38) Noo poy ngee-vichu-ni-q.

I him leave-want-make-Tns

'I made him want to leave.'

Syntactically, it consists of a morphologically complex verb together with a subject and an object pronoun. Semantically, we need to show that the speaker is the logical subject of 'make' (i.e. the one who does the making), while the object 'him' has multiple roles: as the semantic object of 'make' (the target of the agent's force), and as the semantic 
subject of both 'want' and 'leave.'

In (39), I show how the verb stem ngeevichuni 'make want to leave' is progressively assembled. The component elements are ngee 'leave,' -vichu 'want,' and -ni 'make.' The root ngee profiles the trajector's motion away from some original location. The suffix -vichu 'want' designates the trajector's desire (dashed arrow) for the occurrence of some process, which functions as a kind of landmark (a relational landmark rather than a nominal one). Since - vichu is only used for cases where the process desired is one carried out by its trajector, a correspondence internal to its semantic pole identifies the trajector of -vichu with the actor of the landmark process. Finally, -ni profiles an act of causation (double arrow), where the force is directed at a target (a nominal landmark) by way of inducing some occurrence (a relational landmark). The nominal landmark is simultaneously the target of force and the trajector of the schematic process induced.

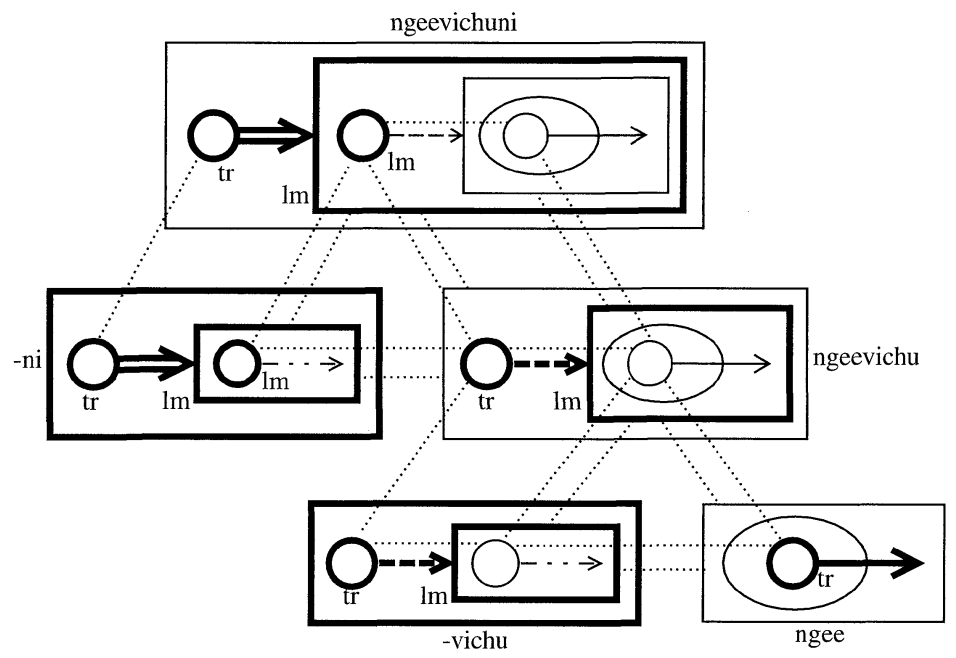

The verb stem is straightforwardly assembled by constructions representing two successive levels of morphological organization. At the lower level, ngee 'leave' specifies the schematic process functioning as the landmark of -vichu 'want,' i.e. the desired event. The composite expression ngeevichu thus profiles the process of the trajector desiring an event consisting of the trajector leaving. At the higher level of organization, ngeevichu specifies the caused occurrence schematically evoked by $-n i$ 'make.' The nominal landmark of $-n i$ is thus identified with the trajector of ngeevichu, i.e. with the person who has the desire 
and the one who does the leaving. At higher levels of organization (not shown), the subject pronoun noo ' $\mathrm{I}$ ' elaborates the trajector, and the object pronoun poy 'him' specifies the landmark. The speaker is thus identified as the causer, and 'him' as the target of force, the wanter, and the leaver.

\section{Constituency}

The foregoing examples suggest how CG handles the complexities of grammatical structure without positing syntactic tree structures. To the extent that it is valid, the information captured in such tree structures is also captured in the CG account based on symbolic assemblies. Three kinds of information are represented in classic generative phrase trees: constituency, linear order, and category membership (via node labels). It is not denied that these kinds of information need to be provided, only that their expression requires a separate, autonomous formal device.

Given that basic grammatical categories have conceptual characterizations, and that an expression's category is determined by the nature of its profile, category information is inherent in each symbolic structure in an assembly, as an aspect of its semantic structure. In (37), for example, throw is a verb because it profiles a process, rock is a noun because it profiles a thing, and into a preposition because it profiles a non-processual relationship with a thing as landmark. Higher level categories are also susceptible to semantic or symbolic characterization. A full noun phrase - what I call a nominal-profiles a thing which is grounded, i.e. a grammaticized indication is given of its relation to the speech situation. The composite expressions a rock and the pond are thus identified as nominals by virtue of being grounded by the indefinite and definite articles. Similarly, a prepositional phrase can be characterized as a symbolic assembly in which a nominal elaborates the preposition's landmark. Into the pond is thus a prepositional phrase (though I have not shown the full assembly in (37)).

Linear order is actually temporal order in the speech stream. As such, this aspect of symbolic assemblies is specified at the phonological pole of each symbolic structure. In (12), for instance, the composite phonological structure of smart woman indicates that the word symbolizing the adjective directly precedes the word symbolizing the noun ( $T=$ processing time). Importantly, this dimension of phonological 
structure, exploited for grammatical (symbolizing) purposes, is given as part of the internal structure of each symbolic element. It is not essential that linear order be represented in these diagrams by relative position on the page of the component symbolic structures.

Finally, constituency is a matter of a composite structure in one construction functioning in turn as component structure in another-the order in which simpler symbolic structures are successively integrated to form progressively more elaborate symbolic structures. It is just a matter of hierarchy, a general feature of cognitive organization, even motor routines.

A crucial point is that grammatical constituency pertains to the external arrangement of symbolic structures, taking these as wholes (i.e. as bipolar entities). This external hierarchical arrangement need not coincide with any hierarchical structure evident internally at the semantic or phonological pole of symbolic elements. That is, grammatical constituency need not parallel any purely conceptual hierarchy manifested in the semantic pole of component or composite symbolic structures, nor any purely phonological hierarchy manifested in their phonological poles. Internally, at each pole, any hierarchical arrangement is determined locally, by factors that are intrinsically conceptual or intrinsically phonological (hence unipolar-see Langacker (1987a: 2.3.1.2)).

For example, in casual pronunciation the definite article cliticizes to the following word, with which it forms a single intonational unit. A nominal such as the smart woman therefore shows the intrinsically phonological organization indicated in (40a). Semantically, however, the article does not pertain to the adjective, but to the entire nominal expression. For semantic and grammatical purposes, we want to say that it combines with the remainder of the nominal as the last step in assembling it, implying the constituency in (40b).

(40) a. (th'smart) (woman)

[unipolar (purely phonological) constituency]

b. (the) (smart woman)

[bipolar (grammatical) constituency]

This apparent discrepancy is unproblematic given the distinction between grammatical hierarchy - pertaining to the external arrangement of symbolic structures as bipolar entities - and the phonological hierarchy internal to each symbolic structure at its phonological pole, viewed as a purely phonological (unipolar) entity. Unipolar phonological structure involves the grouping of segments into syllables, syllables into 
words, words into phonological phrases, etc. The phonological elements directly relevant to grammar are those defined in bipolar terms, i.e. through their participation in symbolic relationships. We should not expect that these will always coincide with natural phonological units (e.g. morpheme boundaries do not necessarily coincide with syllable boundaries), nor that the two will mirror one another in their hierarchical arrangements.

Diagrammed in (41) is the phonological pole of th'smart woman, for the normal pronunciation (40a). In terms of grammatical constituency, where phonological elements are delimited by their participation in symbolic relationships, the constituency is the expected one, represented in (40b). At the lower level of organization, smart and woman combine to form smart woman, and then, at the higher level of organization, the definite article combines with this to form the full nominal. In casual speech, the definite article cliticizes to the following word. I represent its clitic status by showing the consonant th as combining with a word to form a higher level word. Of course, internally to the definite article the word it combines with is specified only schematically. In the construction, this word is put in correspondence with the first word of the other component structure, in this case smart. The composite phonological structure is thus as given in (40a).

(41)

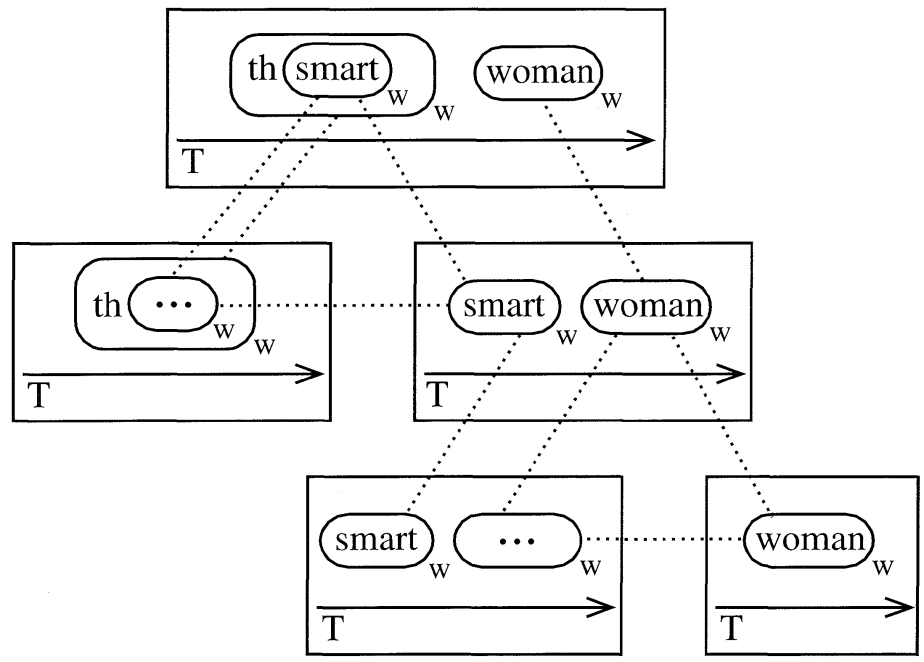

The expression's semantic pole is sketched in (42). Grammatically, i.e. in terms of the external relationships between semantic structures, woman and smart combine at the lower level of constituency, with 
smart woman then combining with the at the higher level. Internally, the semantic structures of smart, woman, and smart woman are just as shown in previous diagrams. As for the article, the contextual definiteness it conveys is represented by dashed arrows indicating that the speaker (S) and hearer $(\mathrm{H})$ are able to direct their attention to the same focused entity, characterized schematically as a thing. In the higher level construction, this thing functions as an e-site. It corresponds to the profile of smart woman, which elaborates it.

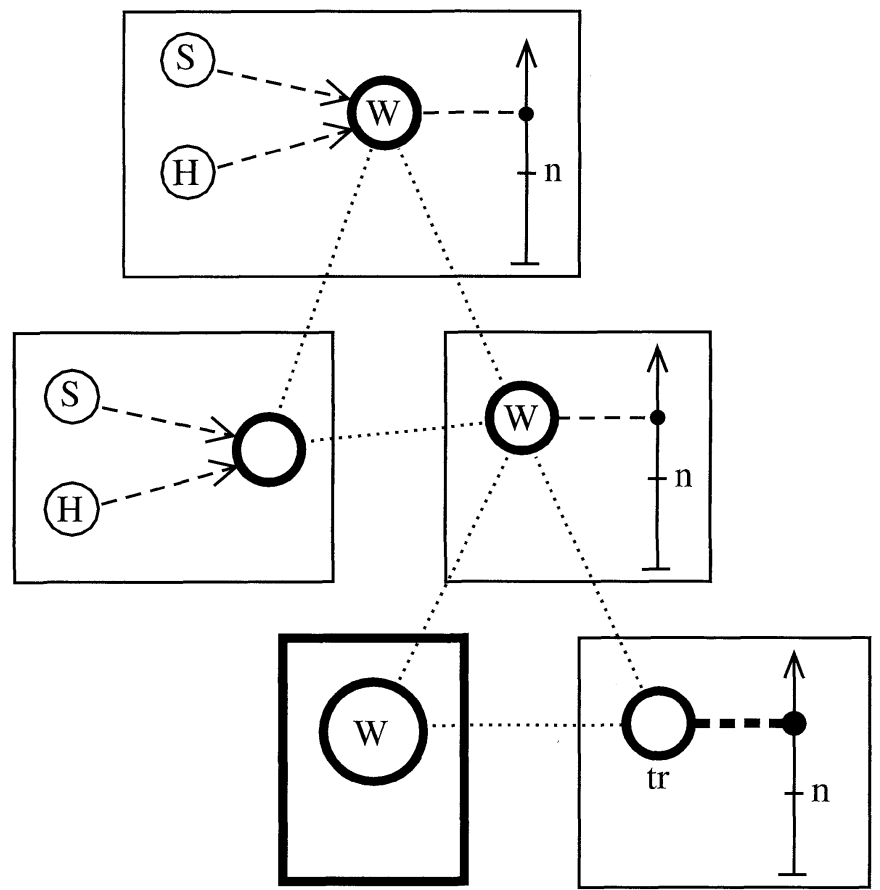

How can the definite article pertain to the noun woman in particular when it combines grammatically with the composite expression smart woman? The answer is that smart woman, despite its grammatical complexity, profiles the woman at the composite structure level. That is, the woman is the conceptual referent of the composite expression, just as it is for woman individually. Since profiling determines grammatical category, the composite expression smart woman is itself a complex noun. The definite article can thus combine with it by virtue of a correspondence between their profiles, just as it can with a simple noun. In short, the grammatical constituency, where smart and woman are on a par, is not isomorphic to the internal conceptual orga- 
nization at the composite structure's semantic pole, where only the woman stands in profile.

A clearer, more striking example is provided by complementation. A complex sentence like (43a), with two levels of embedding, would normally be pronounced with the intonation shown, where pauses occur between each clause. However, it would generally be analyzed syntactically as shown in (43b). The intonation suggests a flat structure, with no grouping of clauses, whereas the presumed grammatical constituency is hierarchical, defined by two levels of clausal embedding. To reconcile the flat intonational organization with the hierarchical grammatical structure, theorists have sometimes posited special devices such as phonological readjustment rules (Chomsky and Halle (1968: 6.5)).

(43) a. Bill said/Joe believes/Roger is angry.

b. [Bill said [Joe believes [Roger is angry]]]

I suggest that the standard syntactic analysis, engendering the need for readjustment, confuses grammatical constituency with hierarchical organization that is purely conceptual in nature. From the CG perspective, nothing precludes a grammatical description which directly accommodates the apparently flat structure observed in (43a). The embedding shown in (43b) is ultimately a matter of conceptual embedding, in which one mental space is embedded in another (Fauconnier $(1985,1997))$. Both say and believe are space builders, each setting up a mental space representing the content of the statement or the belief. As the content of Joe's belief, Roger's being angry is conceptually embedded as one participant of believe. Likewise, as the content of Bill's statement, Joe's belief in regard to Roger is conceptually embedded as one participant of say. This conceptual layering of mental spaces is shown in (44) as the composite semantic structure of the entire expression. However, this conceptual structure-the semantic pole of the complex sentence-need not mirror the external relationships of symbolic structures defining grammatical constituency. 
(44)

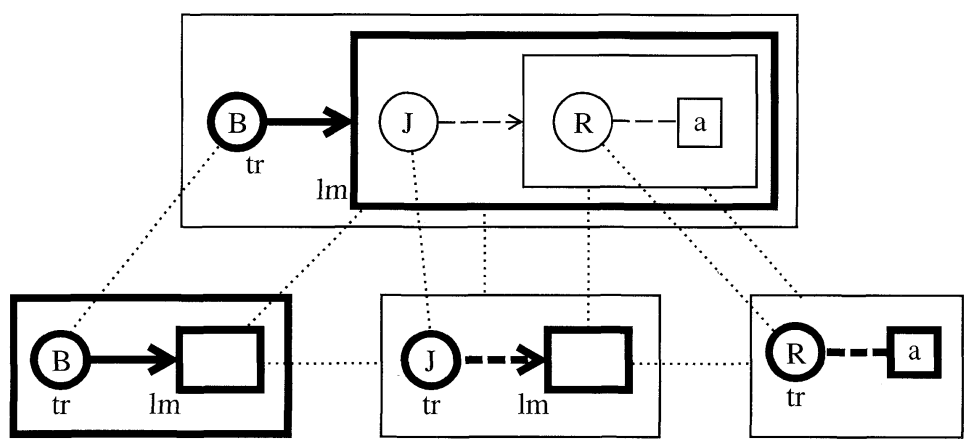

Diagram (44) shows how, assuming a flat constituency, the three clauses are directly integrated to form the composite expression. The first two clauses, Bill said and Joe believes, exhibit subject+verb constituency. Their landmarks are schematic propositions, each elaborated by the finite clause that follows. Within this flat structure, the initial clause functions as profile determinant, since the overall expression designates an act of saying (not believing or being angry). So far as I can tell, all the necessary semantic and grammatical relationships are captured, despite the flat constituency. The evident hierarchy is conceptual in nature, the successive embedding of mental spaces. It is the same regardless of the order in which the component finite clauses are integrated, provided that the proper correspondences are established.

From considerations like these, I conclude that grammatical constituency is less central, and its role more limited, than is commonly assumed. When it is properly distinguished from purely conceptual and purely phonological organization, and when the pivotal role of correspondences is recognized, grammatical constituency can be seen as non-essential and often variable. It is not fundamental, but emerges as a special case from other, more basic phenomena (Langacker (1995, 1997)).

\section{Conclusion}

The question is often posed whether grammar is predictable from semantics and other independent factors. From a negative answer to that question, it is generally concluded that grammar constitutes an autonomous formal system. I suggest, however, that this conclusion is unwarranted, if only because the reasoning fails to distinguish between strong and weak autonomy. The non-predictability of grammar, i.e. 
weak autonomy, does not itself determine what kinds of units are needed to describe it. In particular, it does not establish the claim of strong autonomy: that grammar is distinct from both lexicon and semantics, constituting a separate level of representation whose description requires a special set of irreducible grammatical primitives. I have outlined an alternative view which accommodates weak autonomy while denying strong autonomy. In CG, grammar and lexicon form a continuum fully describable as assemblies of symbolic structures (formmeaning pairings). On this account, it makes no sense to ask whether grammar is predictable from meaning, since grammar incorporates meaning, as one of its two poles. The question is analogous to asking whether the phonological shape of a lexical item can be predicted from its semantic value. While the answer is clearly negative, it does not establish the independence of lexicon from semantics. A dictionary listing only lexical forms, without their meanings, would not be very interesting.

A more reasonable question to pose is why grammar should exist in the first place. I can best conclude by quoting a previous answer (Langacker (1999b: 55)):

Grammar would not exist if lexical units were available to symbolize every conception we wanted to express. But they are not. Lexical units form a limited set, while the conceptions we want to encode linguistically are open-ended and indefinitely varied. To overcome this, we resort to complex expressions consisting of multiple lexical elements. Each lexical component evokes some facet of the overall conception, one singled out precisely by virtue of being susceptible to individual symbolization. Collectively, these individually symbolized conceptual chunks give enough information about the composite conception intended by the speaker that the addressee, in context, is able to reconstruct some approximation to it. However, this reconstruction requires some indication of how the conceptual chunks are supposed to fit together. The role of grammar is to provide this information. 


\section{REFERENCES}

Chomsky, Noam and Morris Halle (1968) The Sound Pattern of English, Harper \& Row, New York.

Croft, William (2001) Radical Construction Grammar: Syntactic Theory in Typological Perspective, Oxford University Press, Oxford.

Fauconnier, Gilles (1985) Mental Spaces: Aspects of Meaning Construction in Natural Language, MIT Press/Bradford, Cambridge, MA and London.

Fauconnier, Gilles (1997) Mappings in Thought and Language, Cambridge University Press, Cambridge.

Fillmore, Charles J. (1988) "The Mechanisms of "Construction Grammar," BLS 14, 35-55.

Fillmore, Charles J., Paul Kay, and Mary Catherine O'Connor (1988) "Regularity and Idiomaticity in Grammatical Constructions: The Case of Let Alone," Language 64, 501-538.

Goldberg, Adele E. (1995) Constructions: A Construction Grammar Approach to Argument Structure, University of Chicago Press, Chicago and London.

Hudson, Richard A. (1987) "Zwicky on Heads," Journal of Linguistics 23, 109-132.

Langacker, Ronald W. (1973) "Predicate Raising: Some Uto-Aztecan Evidence," Issues in Linguistics: Papers in Honor of Henry and Renée Kahane, ed. by Braj B. Kachru et al., 468-491, University of Illinois Press, Urbana.

Langacker, Ronald W. (1987a) Foundations of Cognitive Grammar, vol. 1, Theoretical Prerequisites, Stanford University Press, Stanford.

Langacker, Ronald W. (1987b) "Nouns and Verbs," Language 63, 53-94.

Langacker, Ronald W. (1988a) "A Usage-Based Model," Rudzka-Ostyn, 127-161.

Langacker, Ronald W. (1988b) "The Nature of Grammatical Valence," Rudzka-Ostyn, 91-125.

Langacker, Ronald W. (1990) Concept, Image, and Symbol: The Cognitive Basis of Grammar, Mouton de Gruyter, Berlin and New York.

Langacker, Ronald W. (1991) Foundations of Cognitive Grammar, vol. 2, Descriptive Application, Stanford University Press, Stanford.

Langacker, Ronald W. (1993a) "Universals of Construal," BLS 19, 447-463.

Langacker, Ronald W. (1993b) "Grammatical Traces of Some 'Invisible' Semantic Constructs," Language Sciences 15, 323-355.

Langacker, Ronald W. (1993c) "Reference-Point Constructions," Cognitive Linguistics 4, 1-38.

Langacker, Ronald W. (1995) "Conceptual Grouping and Constituency in Cognitive Grammar," Linguistics in the Morning Calm 3, ed. by Ik-Hwan Lee, 149-172, Hanshin, Seoul.

Langacker, Ronald W. (1997) "Constituency, Dependency, and Conceptual Grouping," Cognitive Linguistics 8, 1-32. 
Langacker, Ronald W. (1999a) Grammar and Conceptualization, Mouton de Gruyter, Berlin and New York.

Langacker, Ronald W. (1999b) "Correspondences, Compositionality, and Grammar," Actas del XXI Congreso Internacional de A.E.D.E.A.N. (Asociación Española de Estudios Anglo-Norteamericanos), ed. by Fernando Toda Iglesia et al., 55-74, Secretariado de Publicaciones de la Universidad de Sevilla, Seville.

Langacker, Ronald W. (1999c) "Assessing the Cognitive Linguistic Enterprise," Cognitive Linguistics: Foundations, Scope, and Methodology, ed. by Theo Janssen and Gisela Redeker, 13-59, Mouton de Gruyter, Berlin and New York.

Langacker, Ronald W. (2000) "A Dynamic Usage-Based Model," Usage-Based Models of Language, ed. by Michael Barlow and Suzanne Kemmer, 1-63, CSLI Publications, Stanford.

Langacker, Ronald W. (2001) "Topic, Subject, and Possessor," A Cognitive Approach to the Verb: Morphological and Constructional Perspectives, ed. by Hanne Gram Simonsen and Rolf Theil Endresen, 11-48, Mouton de Gruyter, Berlin and New York.

Langacker, Ronald W. (To appear a) "Context, Cognition, and Semantics: A Unified Dynamic Approach."

Langacker, Ronald W. (To appear b) "Integration, Grammaticization, and Constructional Meaning."

Rudzka-Ostyn, Brygida, ed. (1988) Topics in Cognitive Linguistics, John Benjamins, Amsterdam and Philadelphia.

Vandeloise, Claude (1991) Spatial Prepositions: A Case Study from French, University of Chicago Press, Chicago and London.

Zwicky, Arnold M. (1985) "Heads," Journal of Linguistics 21, 1-29.

Linguistics, 0108

UCSD

La Jolla, CA 92093-0108

USA

e-mail: rlangacker@ucsd.edu 